TRANSACTIONS OF THE

AMERICAN MATHEMATICAL SOCIETY

Volume 358, Number 5 , Pages 1869-1895

S 0002-9947(05)03936-X

Article electronically published on December 20, 2005

\title{
ALGEBRAIC GOODWILLIE CALCULUS AND A COTRIPLE MODEL FOR THE REMAINDER
}

\author{
ANDREW MAUER-OATS
}

\begin{abstract}
Goodwillie has defined a tower of approximations for a functor from spaces to spaces that is analogous to the Taylor series of a function. His $n^{\text {th }}$ order approximation $P_{n} F$ at a space $X$ depends on the values of $F$ on coproducts of large suspensions of the space: $F\left(\vee \Sigma^{M} X\right)$.

We define an "algebraic" version of the Goodwillie tower, $P_{n}^{\text {alg }} F(X)$, that depends only on the behavior of $F$ on coproducts of $X$. When $F$ is a functor to connected spaces or grouplike $H$-spaces, the functor $P_{n}^{\mathrm{alg}} F$ is the base of a fibration

$$
\left|\perp^{*+1} F\right| \rightarrow F \rightarrow P_{n}^{\mathrm{alg}} F,
$$

whose fiber is the simplicial space associated to a cotriple $\perp$ built from the $(n+1)^{\text {st }}$ cross effect of the functor $F$. In a range in which $F$ commutes with realizations (for instance, when $F$ is the identity functor of spaces), the algebraic Goodwillie tower agrees with the ordinary (topological) Goodwillie tower, so this theory gives a way of studying the Goodwillie approximation to a functor $F$ in many interesting cases.
\end{abstract}

\section{INTRODUCTION}

A function on the real line can be approximated by its ordinary Taylor series at a point, with the " $n^{\text {th }}$-order approximation" being the Taylor polynomial through the $x^{n}$ term. Goodwillie [5, 6, 7, has defined an analogous approximation for functors of topological spaces.

Johnson and McCarthy 8 have explored alternative models for Goodwillie's approximation, working in the simpler setting of chain complexes. Specifically, for a functor $C$ that is the prolongation to chain complexes of a functor to an abelian category, they have defined a tower of functors to chain complexes, which we denote $P_{n}^{\text {alg }} C$ to distinguish from Goodwillie's tower. The functor $P_{n}^{\text {alg }}$ approximates $C$ in the sense that the map $C \rightarrow P_{n}^{\text {alg }} C$ is the universal map under $C$ to a functor whose $(n+1)^{\text {st }}$ cross-effect $\perp_{n+1} C$ is acyclic. In a later work $[9$, the same authors show that $P_{n}^{\text {alg }}$ can be constructed from a cotriple. One source of the interest in $P_{n}^{\text {alg }}$ approximation stems from the fact that, for many functors, the cross effects are more computationally accessible than the stabilization; hence, $P_{n}^{\text {alg }}$ should also be more accessible than Goodwillie's $P_{n}$.

Taking their work as inspiration, we explore to what extent similar ideas are useful in the study of functors from spaces to spaces. In this context, we develop a tower analogous to that of Johnson and McCarthy. We call this an algebraic Goodwillie tower, and denote it $P_{n}^{\text {alg }} F(X)$. The algebraic Goodwillie approximation was

Received by the editors December 9, 2002.

2000 Mathematics Subject Classification. Primary 55P65.

(C)2005 American Mathematical Society Reverts to public domain 28 years from publication 1869 
created with the intent that it be universal with respect to natural transformations of $F$ to functors whose $(n+1)^{\text {st }}$ cross effect is contractible; however, it turns out that there is a subtle issue involving $\pi_{0}$. For example, the vanishing of the second cross effect forces a monoidal structure on $\pi_{0}$, but on $\pi_{0}$ the approximation process can only produce the group completion of this monoid (since it involves loops on a space). We solve this problem by requiring our functors to take values in topological groups or connected spaces.

Our Main Theorem is that for good functors $F$, there exists a fibration sequence up to homotopy

$$
\left|\perp_{n+1}^{*+1} F\right| \rightarrow F \rightarrow P_{n}^{\text {alg }} F
$$

where the fiber is built from a cotriple $\perp$ formed from the diagonal of the $(n+1)^{\text {st }}$ cross effect. In [9], the authors are able to define the analog of $P_{n}^{\text {alg }}$ simply by taking the cofiber of the left-hand map in (1.1). However, in an unstable topological setting, that approach is not useful, and the proof is much more difficult.

It is easy to show that when $F$ commutes with realizations, the $n^{\text {th }}$ algebraic Goodwillie approximation $P_{n}^{\text {alg }} F(X)$ agrees with the $n^{\text {th }}$ topological Goodwillie approximation $P_{n} F(X)$ (see 3.10), so in this case (1.1) shows that the "remainder" of the $n$-excisive Goodwillie approximation is the cotriple homology of $F$.

1.1. Organization of the paper. We state the Main Theorem in $₫ 3$, along with all of the basic definitions necessary to understand it. In 8 , we summarize technical ingredients of the proof, including the limit axiom, $\pi_{*}$-Kan functors, results of Goodwillie, and iterated cross effects. Then $\$ 5$ shows that the approximation $P_{n}^{\text {alg }}$ preserves the connectivity of natural transformations. To deal with another (important) technical issue, $\$ 6$ sketches proofs that when the cross effect vanishes, a certain cube is actually Cartesian (rather than just having contractible total fiber). In \$7, we prove that $\perp$ is really a cotriple. After these technicalities, we give a detailed outline of the Main Theorem ( $(8)$. The proof of the main theorem then follows, with $₫ 9$ proving that when $\perp_{n+1} F(X) \simeq 0$, then $F(X) \simeq P_{n}^{\text {alg }} F(X)$, and $\$ 10$ reducing the general case to that case.

\section{Preliminaries}

The category of "spaces" is taken to be the category of compactly generated spaces with nondegenerate basepoint. The space 0 or $*$ is the space with only one point. When forming the function $\operatorname{space} \operatorname{Map}(A, B)$, we always implicitly replace $A$ by a cofibrant approximation (CW complex) and $B$ by a fibrant approximation (which is no change with our definition of spaces). When forming the geometric realization, we first "thicken" each space ([10, p. 308]) so the resulting functor has good homotopy behavior. When we say two spaces are equivalent, we mean they are weakly homotopy equivalent. We use the term fibration sequence up to homotopy for a sequence $F \rightarrow E \rightarrow B$ in which $F \stackrel{\simeq}{\rightarrow} \operatorname{hofib}(E \rightarrow B)$.

We briefly recall a few standard properties of functors. A functor is continuous if the map $\operatorname{Map}(X, Y) \rightarrow \operatorname{Map}(F(X), F(Y))$ sending $f$ to $F(f)$ is continuous. A functor is a homotopy functor if it preserves weak homotopy equivalences. A functor is called reduced if $F(0) \simeq 0$. 
Definition 2.1. A homotopy functor $F$ is said to satisfy the limit axiom if $F$ commutes with filtered homotopy colimits of finite complexes. That is, if hocolim $F\left(X_{\alpha}\right)$ $\stackrel{\simeq}{\longrightarrow} F\left(\right.$ hocolim $\left.X_{\alpha}\right)$ for all filtered systems $\left\{X_{\alpha}\right\}$ of finite complexes.

In this paper, we assume that all functors are continuous homotopy functors that satisfy the limit axiom. All functors are defined from pointed spaces to pointed spaces unless otherwise stated.

Cubical diagrams and homotopy fibers figure heavily into this work, so we recall several definitions from [6]. Let $T$ be a finite set. $\mathcal{P}(T)$ is the poset of subsets of $T$ (regarded as a category). $\mathcal{P}_{0}(T)$ is the poset of nonempty subsets of $T$. A $T$-cube is a functor defined on $\mathcal{P}(T)$. If $\mathcal{X}$ is a $T$-cube of pointed spaces, then its homotopy fiber, hofib $\mathcal{X}$, is the subspace of the function space $\prod_{U \subset T} \operatorname{Map}\left([0,1]^{U}, \mathcal{X}(U)\right)$ consisting of maps that are natural in $U$ and that send points with any coordinate 1 to the basepoint. A formal definition is given in [6, 1.1]. Alternatively, the homotopy fiber can be constructed by iterating the process of taking fibers in a single direction.

We write $\mathbf{n}$ for the finite (unpointed) set $\{1, \ldots, n\}$, and $[n]$ for the finite space $\bigvee^{n} S^{0} \cong\{0, \ldots, n\}$, with basepoint 0 .

\section{The Main Theorem}

In this section, we briefly present all of the background necessary to understand the Main Theorem (3.13). In brief: cross effects, left Kan extensions, excisive functors, and $P_{n}^{\text {alg }}$.

The cross effect measures the failure of a functor to take coproducts to products.

Definition $3.1\left(c r_{n}\right)$. Define the $n^{\text {th }}$ cross-effect cube, $\mathcal{C} \mathcal{R}_{n} F\left(X_{1}, \ldots, X_{n}\right)$, to be the n-cube $\mathcal{X}$ with $\mathcal{X}(U)=F\left(\bigvee_{u \notin U} X_{u}\right)$ and $\mathcal{X}(i: U \rightarrow V)$ induced by the identity of $X_{u}$ if $u \notin V$ and the map to the basepoint if $u \in V$.

Let $c r_{n} F\left(X_{1}, \ldots, X_{n}\right)$ denote the homotopy fiber of $\mathcal{X}$.

Definition $3.2\left(\perp_{n}, \epsilon\right)$. Let $\mathcal{X}=\mathcal{C R}_{n} F(X, \ldots, X)$ and let $\mathcal{Y}$ be the $\mathbf{n}$-cube with

$$
\mathcal{Y}(U)= \begin{cases}F(X) & \text { if } U=\emptyset \\ * & \text { otherwise. }\end{cases}
$$

Define $\perp_{n} F(X)=$ hofib $\mathcal{X}$, and note that $F(X) \cong$ hofib $\mathcal{Y}$. Using the fold map $\bigvee X \rightarrow X$ on the vertex $U=\emptyset$ and the zero map elsewhere induces a map of cubes $\mathcal{X} \rightarrow \mathcal{Y}$. Define $\epsilon$ to be the induced map on homotopy fibers, so

$$
\epsilon: \perp_{n} F(X) \rightarrow F(X) .
$$

As we will show in Section $7, \perp_{n}$ is part of a cotriple. Hence there is a simplicial object $Y$ with $Y_{k}=\perp_{n}^{k+1} F(X)$ and face maps $d_{i}^{(k)}=\perp_{n}^{i} \epsilon \perp_{n}^{k-i}$.

Example 3.3. The second cross effect of the functor $F(X)=Q(X)$ is contractible, but the second cross effect of $F(X)=Q(X \wedge X)$ is

$$
c r_{2} F(X, Y) \simeq Q(X \wedge Y) \times Q(Y \wedge X) .
$$

The left Kan extension gives a canonical way of extending a functor from a subcategory to a functor defined on the whole category.

Definition 3.4 (left Kan extension). Let $L F$ denote the homotopy invariant left Kan extension of a functor $F$ along the inclusion of finite pointed sets into pointed 
spaces. Letting $\mathcal{S}$ denote a small category of finite pointed sets and $\mathcal{T}$ denote the category of pointed spaces, the realization of the following simplicial space can taken to be the definition of $\operatorname{LF}(-)$ :

$$
[n] \mapsto \bigvee_{\left(C_{0}, \ldots, C_{n}\right)} F\left(C_{0}\right) \wedge\left(\operatorname{Map}_{\mathcal{S}}\left(C_{0}, C_{1}\right) \times \cdots \times \operatorname{Map}_{\mathcal{T}}\left(C_{n},-\right)\right)_{+}
$$

The coproduct is taken over all $\left(C_{0}, \ldots, C_{n}\right) \in \mathcal{S}^{\times n}$.

When $F$ is the restriction of a functor (also called $F$ ) defined on $\mathcal{T}$, then there is a natural map $a: L F(Y) \rightarrow F(Y)$ induced by

$$
F\left(C_{0}\right) \wedge \operatorname{Map}\left(C_{0}, Y\right)_{+} \rightarrow F\left(C_{0}\right) \wedge \operatorname{Map}\left(F\left(C_{0}\right), F(Y)\right)_{+} \rightarrow F(Y)
$$

sending

$$
y \wedge f \mapsto y \wedge F(f) \mapsto F(f)(y)
$$

We have required that all functors be continuous to guarantee that the first map above is continuous.

Lemma 3.5. When $Y$ is a finite pointed set in $\mathcal{S}$, the map a: $L F(Y) \rightarrow F(Y)$ is a simplicial homotopy equivalence.

Proof. When $Y \in \mathcal{S}$, the category $\mathcal{S} \downarrow Y$ has a terminal object. This immediately translates into a homotopy contracting $L F(Y)$ to $F(Y) \wedge\left(i d_{Y}\right)_{+} \cong F(Y)$.

When working with the left Kan extension, we will frequently want to shift the functor so that we can examine its value on coproducts of spaces other than $S^{0}$. To do this, we write $F_{X}(-)$ for the functor $F(X \wedge-)$.

We primarily understand $L F$ as " $F$ made to commute with realizations", in the sense of the following lemma.

Lemma 3.6. Let $Y$. be a simplicial set. Then

$$
L F_{X}(|Y .|) \simeq\left|F_{X}(Y .)\right|=|F(X \wedge Y .)|
$$

Proof. Using (3.1), this follows from the observation that $\operatorname{Map}(S, Y) \cong \prod_{s \in S} Y$ commutes with realizations when $S$ is a finite set.

Before we define the algebraic Goodwillie tower, we will recall the classical definition of the topological Goodwillie tower.

Definition 3.7 (Cartesian, co-Cartesian). An $S$-cube $\mathcal{X}$ is Cartesian if the categorical map $\mathcal{X}(\emptyset) \cong \operatorname{holim}_{\mathcal{P}(S)} \mathcal{X} \rightarrow \operatorname{holim}_{\mathcal{P}_{0}(S)} \mathcal{X}$, induced by the inclusion of the category $\mathcal{P}_{0}(S)$ of nonempty subsets of $S$ into the category $\mathcal{P}(S)$ of all subset of $S$, is a weak equivalence. An $S$-cube $\mathcal{X}$ is co-Cartesian if the categorical map $\operatorname{hocolim}_{\mathcal{P}_{1}(S)} \mathcal{X} \rightarrow$ hocolim $_{\mathcal{P}(S)} \mathcal{X} \cong \mathcal{X}(S)$, induced by the inclusion of all proper subsets of $S$ into all subsets of $S$, is a weak equivalence. An $S$-cube is strongly co-Cartesian if every sub-2-cube is co-Cartesian.

Definition 3.8 ( $n$-excisive [6, 3.1]) $F$ is $n$-excisive if for every strongly co-Cartesian $(n+1)$-cube $\mathcal{X}$, the cube $F \mathcal{X}$ is Cartesian. 
In [7, Goodwillie constructs a universal $n$-excisive approximation $P_{n} F$ to a functor $F$. The approximations form a tower of functors equipped with natural transformations of the following form:

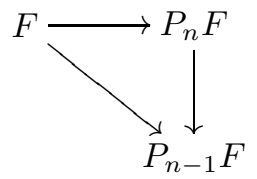

We are now in a position to define the algebraic Goodwillie approximation.

Definition $3.9\left(P_{n}^{\mathrm{alg}} F\right)$. The algebraic Goodwillie approximation $P_{n}^{\text {alg }} F(X)$ is defined to be the functor given by applying $P_{n}$ to the left Kan extension of $F$ shifted over $X$; that is, $P_{n}^{\text {alg }} F(X)=P_{n}\left(L F_{X}\right)\left(S^{0}\right)$.

The natural transformation $F(X) \rightarrow P_{n}^{\text {alg }} F(X)$ arises from evaluating the map $L F_{X} \rightarrow P_{n}\left(L F_{X}\right)$ at $S^{0}$.

Proposition 3.10. If $F_{X}$ is a functor that commutes with realizations, that is, the natural map $L F_{X}(Y) \rightarrow F_{X}(Y)$ is an equivalence for all spaces $Y$, then the natural map $P_{n}^{\text {alg }} F(X) \rightarrow P_{n} F(X)$ is an equivalence.

Proof. Given $L F_{X} \stackrel{\simeq}{\longrightarrow} F_{X}$, applying $P_{n}$ and evaluating at $S^{0}$ gives $P_{n}\left(L F_{X}\right)\left(S^{0}\right) \stackrel{\simeq}{\longrightarrow}$ $P_{n} F_{X}\left(S^{0}\right)$. The left-hand side is $P_{n}^{\operatorname{alg}} F(X)$, and the right-hand side is $P_{n} F(X)$.

There remains a technical hypothesis on $F$, related to the " $\pi_{*}$-Kan condition", needed in the Main Theorem. We give the hypotheses here, but defer discussion of how they are used until Section 4.3 .

Hypothesis 3.11 (Connected Values). $F$ has connected values (on coproducts of $X$ ) if the functor $L F_{X}$ is always connected.

Let $\mathcal{G}$ denote the category of grouplike $H$-spaces. Specifically, by $\mathcal{G}$, we mean the category of algebras over the associativity operad with inverses and identity up to homotopy. In this category, all morphisms strictly preserve all homotopies, so it is rigid enough that the realization of a simplicial object in $\mathcal{G}$ is still in $\mathcal{G}$.

Hypothesis 3.12 (Group Values). In the following definition, let $\mathcal{T}$ denote the category of pointed spaces. Let $U: \mathcal{G} \rightarrow \mathcal{T}$ be the forgetful functor.

$F$ "has group values" or "takes values in groups" if there exists a functor $F^{\prime}$ so that the following diagram commutes:

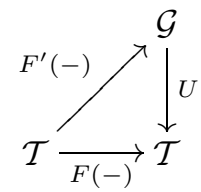

In this case, we will conflate $F(-)$ with its lift to groups. We say that $F$ has group values on coproducts of $X$ if $F_{X}$ has group values.

We are now able to state our theorem relating the cross effects of a functor and the algebraic Goodwillie approximation. 
Theorem 3.13 (Main Theorem). Let $F$ be a reduced homotopy functor from pointed spaces to pointed spaces. If $F$ has either connected values (3.11) or group values (3.12) on coproducts of $X$, then the following is a fibration sequence up to homotopy:

$$
\left|\perp_{n+1}^{*+1} F(X)\right| \rightarrow F(X) \rightarrow P_{n}^{a l g} F(X),
$$

and furthermore the right-hand map is surjective on $\pi_{0}$.

To establish this theorem, we use a "ladder" induction on $n$, where there are two cases for each $n$ : the first depends on the second for smaller $n$, and the second depends on the first for the same $n$. The first case is to establish that when $\perp_{n+1} F$ vanishes - that is, when $F$ is degree $n$ - the map $F \rightarrow P_{n}^{\text {alg }} F$ is actually an equivalence. This proof proceeds by examining a fibration sequence obtained from the second case for smaller $n$. The second case is to consider the general case, in which $\perp_{n+1} F$ may be nonvanishing, and proceed by applying the first case (for the same $n$ ) to the fiber of the map from $F \rightarrow P_{n}^{\text {alg }} F$. A more extensive outline of the proof is given in Section 8 .

\section{TeChnical CONDitions}

In this section, we address many technical aspects needed to make our machinery work.

4.1. Limit axiom. It is of primary importance to know that the functors we will be working with satisfy the limit axiom.

Lemma 4.1. For any functor $F$, the functor $L F$ satisfies the limit axiom (2.1).

Proof. The functor $\operatorname{Map}(K,-)$ satisfies the limit axiom for any compact $K$. Examining (3.1), we see that this implies that $L F$ satisfies the limit axiom.

4.2. Eilenberg-Zilber. An "Eilenberg-Zilber"-type theorem for bisimplicial sets provides a source for connectivity estimates.

Lemma 4.2. Let $X$. and $Y$. be simplicial spaces satisfying the $\pi_{*}-$ Kan condition, and let $f$. $: X . \rightarrow Y$. be a map between them. If $n \geq 0$ and $w \geq-1$ are integers such that for $i<n$, the map $f_{i}$ is a weak equivalence, and for $i \geq n$, the map $f_{i}$ is $w$-connected, then $|f$.$| is (n+w)$-connected.

Proof. Using the homotopy spectral sequence of [2, Theorem B.5], we have a spectral sequence $E_{*, *} \Rightarrow \pi_{*} \mid X$.| and a spectral sequence $F_{*, *} \Rightarrow \pi_{*} \mid Y$. $\mid$, with an $f$ inducing a map of spectral sequences $E \rightarrow F$. The hypotheses imply that $E_{i, j}^{1} \cong F_{i, j}^{1}$ when $i<n$ or $j<w$, and $E_{i, w}^{1}$ surjects onto $F_{i, w}^{1}$. An easy analysis of possible differentials then shows that the map $E_{i, j}^{\infty} \rightarrow F_{i, j}^{\infty}$ is an isomorphism when $i+j<n+w$, and a surjection when $i+j=n+w$.

Corollary 4.3 (Eilenberg-Zilber connectivity estimate). Let $X$ and $Y$ be $\pi_{*}$-Kan functors from $\left(\Delta^{o p}\right)^{\times N}$ to spaces, and let $p=\left(p_{1}, \ldots, p_{N}\right) \in\left(\Delta^{o p}\right)^{\times N}$ denote an index for these multisimplicial spaces. Let $f: X \rightarrow Y$. Suppose that $f(p)$ is $w$ connected for all indices $p$. If in addition, there are integers $\left\{n_{i} \mid i=1, \ldots, N\right\}$ so that $f(p)$ is an equivalence if there exists an $i$ with $1 \leq i \leq N$ such that $p_{i}<n_{i}$, then $|f|$ is $\left(\Sigma n_{i}+w\right)$-connected.

Proof. Iterating Lemma 4.2 produces this result. 
4.3. The $\pi_{*}$-Kan condition. For us to be able to say anything useful about $L F$, we need to know that the Kan extension of the fiber of a map is the fiber of the Kan extensions, and that one can continue to say similar things about the functor defined by taking fibers of certain maps.

Definition 4.4 ( $\pi_{*}$-Kan functor). A functor $F$ is called a $\pi_{*}$-Kan functor if given a map of simplicial sets $p: Y$. $\rightarrow Z$. with a section:

(1) the simplicial spaces $F\left(Y\right.$.) and $F\left(Z\right.$.) satisfy the $\pi_{*}$-Kan condition;

(2) $\pi_{0} F(p$.) is a fibration of simplicial sets; and

(3) as a functor of $p: Y \rightarrow Z$, the fiber of $F(p)$ is still a $\pi_{*}$-Kan functor.

This is useful in practice due to the following theorem of Bousfield and Friedlander, restated for simplicial spaces.

Theorem 4.5 ([2, Theorem B.4]). Let A, B, X, and $Y$ be simplicial spaces, and suppose that the cube

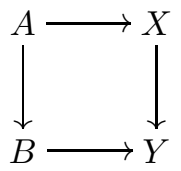

has the property that evaluation at every $[n] \in \Delta^{o p}$ produces a Cartesian cube. If $X$ and $Y$ satisfy the $\pi_{*}$-Kan condition and if $\pi_{0} X \rightarrow \pi_{0} Y$ is a fibration of simplicial sets, then after realization the cube is still Cartesian.

In this work we restrict ourselves to functors satisfying the hypotheses of connected values (3.11) or group values (3.12) so that the simplicial spaces involved always satisfy the $\pi_{*}$-Kan condition. The $\pi_{*}$-Kan condition is a technical fibrancy condition introduced in [2, §B.3] that we do not repeat here.

Corollary 4.6. If $F$ is a $\pi_{*}$-Kan functor, then given a map $p: X \rightarrow Y$ with a section, the spaces $L$ hofib $F(p)$ and hofib $L F(p)$ are equivalent. In view of Lemma 3.6, this is implies $\mid$ hofib $F(p) \mid. \simeq$ hofib $F(|p|$.$) .$

Proof. By definition, a $\pi_{*}$-Kan functor causes $F(X.) \rightarrow F(Y$.) to satisfy the hypotheses on the right-hand vertical map in Theorem 4.5, so letting $B=*$ and $A=$ hofib $F(p$. $)$ produces the desired result.

Remark 4.7. Theorem 4.5 implies that if each space $X_{i}$ is connected, then $\left|\Omega X_{i}\right| \simeq$ $\Omega\left|X_{i}\right|$.

Lemma 4.8. If either $F$ has connected values (3.11) or group values (3.12), then $F$ is a $\pi_{*}$-Kan functor (4.4).

Proof. In these cases, $L F$ always satisfies the $\pi_{*}$-Kan condition ([2, p. 120]). Also, for any surjective map $p$, the function $\pi_{0} F(p$. ) is a fibration of simplicial sets, since surjective maps of simplicial groups are fibrations. If $F$ has connected values, then the requirement that $p$ have a section implies, using the long exact sequence on homotopy, that hofib $F(p)$ is also a functor to connected spaces. If $F$ has group values, then the fact that taking products commutes with taking fibers means hofib $F(p)$ still has group values.

The cross effect of the Kan extension of a $\pi_{*}$-Kan functor can be computed from finite sets, as the following lemma shows. 
Lemma 4.9. Let $Y_{1}, \ldots, Y_{n}$ be spaces. If $F$ is a $\pi_{*}$-Kan functor, then

$$
c r_{n}(L F)\left(Y_{1}, \ldots, Y_{n}\right) \simeq L^{n}\left(c r_{n} F\right)\left(Y_{1}, \ldots, Y_{n}\right),
$$

where $L^{n}$ indicates that the Kan extension is taken in each of the $n$ variables of $c r_{n} F$. The statement above can be abbreviated to $\perp_{n}(L F)(Y) \simeq L\left(\perp_{n} F\right)(Y)$.

Proof. All of the maps in $\mathcal{C R}_{n}(L F)$ have sections, so the hypothesis that $F$ is a $\pi_{*}$-Kan functor means that Corollary 4.6 applies, so taking fibers in one direction commutes with realizations. The sections are all coherent, so they produce a section on the fibers. The property of being a $\pi_{*}$-Kan functor passes to the fibers (by Definition 4.4(3)), so the above argument applies inductively.

Lemma 4.10. Let $\perp=\perp_{n}$ for some $n$. Let $F$. be a simplicial object in the category of either functors to connected spaces or functors to $\mathcal{G}$ (the category of "grouplike $H$-spaces"; see definition prior to 3.12$)$. Then $|\perp F| \stackrel{\simeq}{\longrightarrow} \perp|F|$.

Proof. As in Lemma 4.9, the question is whether the $\perp$ construction may be taken dimensionwise. By the same reasoning as in Lemma 4.8, the spaces in the cube $\mathcal{C} \mathcal{R}_{n} F(X, \ldots, X)$ satisfy the $\pi_{*}$-Kan condition, and on $\pi_{0}$ the maps are fibrations of simplicial sets, so the fibers may be taken dimensionwise. Furthermore, as in that lemma, the cube of fibers has the same property, so we may proceed inductively.

4.4. Goodwillie calculus: Classification of homogeneous functors. The functor $P_{n}$ gives the universal $n$-excisive approximation to a functor. The functor $D_{n}$ gives the homogeneous $n$-excisive part of a functor; it is part of a fibration sequence

$$
D_{n} \rightarrow P_{n} \rightarrow P_{n-1}
$$

Goodwillie shows that there is actually a functorial delooping of the derivative, so this fibration sequence can be delooped once:

Theorem 4.11 ([7, Lemma 2.2]). If $F$ is a reduced homotopy functor from spaces to spaces, then the map $P_{n} F \rightarrow P_{n-1} F$ is part of a fibration sequence

$$
P_{n} F \rightarrow P_{n-1} F \rightarrow \Omega^{-1} D_{n} F,
$$

where $\Omega^{-1} D_{n} F$ is a homogeneous n-excisive functor.

Definition 4.12 (Derivative of $F$ ). The $n^{\text {th }}$ derivative of $F($ at $*)$, denoted $\partial^{(n)} F(*)$, is the following spectrum with $\Sigma_{n}$ action, which we will denote $\mathbf{Y}$. The space $Y_{k}$ in the spectrum is $\Omega^{k(n-1)} \mathrm{cr}_{n} F\left(S^{k}, \ldots, S^{k}\right)$. The structure map $Y_{k} \rightarrow \Omega Y_{k+1}$ arises from suspending inside and looping outside each variable of $c r_{n}$.

When $F$ satisfies the limit axiom (2.1), we can express $D_{n} F(X)$ using the derivative:

Theorem 4.13 ([7, $\S 5$, p. 686]). If $F$ is a homotopy functor from spaces to spaces that satisfies the limit axiom (2.1), then the functor $D_{n} F$ is given by

$$
D_{n} F(X) \simeq \Omega^{\infty}\left(\partial^{(n)} F(*) \wedge_{h \Sigma_{n}} X^{\wedge n}\right),
$$

where smashing over $h \Sigma_{n}$ denotes taking homotopy orbits. 
4.5. Iterated cross effects. Classically, cross-effects are defined inductively, by the repeated application of $\mathrm{Cr}_{2}$ in a single variable. With our definition, it is a theorem that applying $c r_{2}$ in a single variable of $c r_{n}$ produces $c r_{n+1}$.

Recall Goodwillie's notation for sub-cubes: given an $S$-cube $\mathcal{W}$ and a subset $A$ of $S, \partial^{A} \mathcal{W}$ denotes the $A$-cube given by restricting $\mathcal{W}$ to subsets of $A$, and $\partial_{A} \mathcal{W}$ denotes the $(S-A)$-cube with $\partial_{A} \mathcal{W}(B)=\mathcal{W}(A \cup B)$.

Lemma 4.14. For $n \geq 2$, there is a natural equivalence

$$
c r_{2}\left(c r_{n} F\left(X_{1}, \ldots, X_{n-1},-\right)\right)\left(X_{n}, X_{n+1}\right) \stackrel{\simeq}{\longrightarrow} c r_{n+1} F\left(X_{1}, \ldots, X_{n+1}\right) .
$$

Proof. Let $S=\mathbf{n} \amalg \mathbf{2}$, and let $T=S-\{1\} \amalg \emptyset$. The $S$-cube $\mathcal{W}$ defining

$$
c r_{2}\left(c r_{n} F\left(X_{1}, \ldots, X_{n-1},-\right)\right)\left(X_{n}, X_{n+1}\right)
$$

is

$$
\mathcal{W}\left[X_{1}, \ldots, X_{n+1}\right](U \amalg V)= \begin{cases}F\left(\bigvee_{v \notin V} X_{v} \vee \bigvee_{u \notin U \cup\{1\}} X_{u+1}\right), & 1 \notin U, \\ F\left(\bigvee_{u \notin U} X_{u+1}\right), & 1 \in U .\end{cases}
$$

Note that the cube used to compute $c r_{n+1} F\left(X_{1}, \ldots, X_{n+1}\right)$ is exactly the $(n+1)$ cube $\partial^{T} \mathcal{W}$; that is,

$$
\text { hofib } \partial^{T} \mathcal{W}=c r_{n+1} F\left(X_{1}, \ldots, X_{n+1}\right) \text {. }
$$

Also, when $1 \in U$, the sub-cube $\mathcal{W}(U \amalg-)$ is a constant cube, so

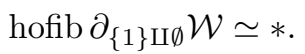

$\mathcal{W}$ can be written as a 1-cube of $(n+1)$-cubes:

$$
\partial^{T} \mathcal{W} \rightarrow \partial_{\{1\} \amalg \emptyset} \mathcal{W}
$$

so the total homotopy fiber of $\mathcal{W}$ is the homotopy fiber of the homotopy fibers of these cubes. Applying (4.1) and (4.2) gives a homotopy fiber sequence

$$
c r_{2}\left(c r_{n} F\left(-, X_{3}, \ldots, X_{n+1}\right)\right)\left(X_{1}, X_{2}\right) \rightarrow c r_{n+1} F\left(X_{1}, \ldots, X_{n+1}\right) \rightarrow *,
$$

so the map from the fiber to the total space is a weak equivalence, as desired.

Corollary 4.15. Suppose that $\perp_{n+1} F \simeq *$. Then $\mathrm{cr}_{2}$ applied in any variable of $c r_{n} F$ results in a contractible functor.

Proof. First, for any spaces $X_{1}, \ldots, X_{n+1}$, the space $c r_{n+1} F\left(X_{1}, \ldots, X_{n+1}\right)$ is a retract of $\perp_{n+1} F\left(X_{1} \vee \cdots \vee X_{n+1}\right) \simeq *$, so $c r_{n+1} F\left(X_{1}, \ldots, X_{n+1}\right)$ is contractible. Lemma 4.14 then shows that $c r_{2}$ applied in any variable of $c r_{n}$ is equivalent to $c r_{n+1}$, and hence contractible.

\section{5. $P_{n}^{\text {ALG }}$ PRESERVES CONNECTIVITY}

In this section, we establish a property of fundamental importance when working with $P_{n}^{\text {alg: }}$ the $n$-additive approximation preserves the connectivity of natural transformations that satisfy some basic good properties. Actually, we prove the slightly stronger result that before evaluation at $S^{0}$, the functor $P_{n} L(-)_{X}$ preserves connectivity.

Theorem 5.1. Let $F$ and $G$ be reduced $\pi_{*}$-Kan functors (4.4). If $\eta: F \rightarrow G$ is a natural transformation that is $w$-connected, then the natural transformation $P_{n}(L \eta)$ is w-connected. 
Once this theorem is established, we have the following immediate corollary.

Corollary 5.2. Let $F$ and $G$ be reduced $\pi_{*}$-Kan functors. If $\eta: F \rightarrow G$ is a natural transformation that is $w$-connected, then the natural transformation $P_{n}^{\text {alg }}(\eta)$ is $w$ connected.

Lemma 5.3. Let $\eta: F \rightarrow G$ be a natural transformation of $\pi_{*}$-Kan functors. If $\eta$ is $w$-connected, then the induced map of derivative spectra $\partial^{(n)} L F(*) \rightarrow \partial^{(n)} L G(*)$ is w-connected.

Proof. Using the Eilenberg-Zilber connectivity estimate (4.3), for all $n \geq 1$, the map $\left|\perp_{n} F\left(S_{.}^{k}\right)\right| \rightarrow\left|\perp_{n} G\left(S_{.}^{k}\right)\right|$ is $(n k+w)$-connected. The derivative spectrum $\partial^{(n)} L F(*)$ then has as its $k^{\text {th }}$ space the space $\Omega^{k(n-1)} \perp_{n} L F\left(S_{.}^{k}\right)$, which by Lemma 4.9 is equivalent to $\Omega^{k(n-1)}\left|\perp_{n} F\left(S^{k}\right)\right|$. On these spaces the map induced by $\eta$ is $(k+w)$-connected, exactly as required to produce a $w$-connected map $\partial^{(n)} L F(*) \rightarrow$ $\partial^{(n)} L G(*)$.

Corollary 5.4. The derivative spectrum $\partial^{(n)} L F(*)$ is connective.

Proof. The map $F \rightarrow 0$ is always 0 -connected.

Corollary 5.5. If $\eta: F \rightarrow G$ is a w-connected map of $\pi_{*}$-Kan functors, then $D_{n}(L \eta)$ is w-connected.

Proof. Recall from Theorem 4.13 that for any functor $H$,

$$
D_{n}(L H)(Y)=\Omega^{\infty}\left(\partial^{(n)} L H(*) \wedge_{h \Sigma_{n}}(Y)^{\wedge n}\right) .
$$

Taking homotopy orbits and smashing with a fixed space preserves connectivity, so this is really a question about the connectivity of the map $\partial^{(n)} L F(*) \rightarrow \partial^{(n)} L G(*)$. The required connectivity was established in Lemma 5.3

Corollary 5.6. If $F$ is a reduced $\pi_{*}$-Kan functor, then the natural transformation $P_{n+1} L F \rightarrow P_{n} L F$ is surjective in $\pi_{0}$.

Proof. Theorem 4.11 (Goodwillie's delooping of $D_{n}$ ) shows that the fibration

$$
D_{n+1} L F \rightarrow P_{n+1} L F \rightarrow P_{n} L F
$$

deloops to a fibration

$$
P_{n+1} L F \rightarrow P_{n} L F \rightarrow \Omega^{-1} D_{n+1} L F .
$$

The delooping of $D_{n+1} L F$ consists of smashing with the suspension of $\partial^{(n+1)} L F(*)$ and taking homotopy orbits. By Corollary 5.4, the spectrum $\partial^{(n+1)} L F(*)$ is connective, so its suspension is 0 -connected. From the long exact sequence on $\pi_{*}$, this implies $P_{n+1} L F \rightarrow P_{n} L F$ is surjective on $\pi_{0}$.

Proof of Theorem 5.1, As above, the spectrum $\partial^{(n+1)} L F(*)$ in Equation (5.1) is connective, so the map on the delooping of $D_{n+1}$ induced by $\eta$ is $(w+1)$-connected. The map on the fibers $\left(P_{n+1}(L \eta)\right)$ is therefore $w$-connected.

A result that is closely related to Theorem 5.1 is the following, which says that $P_{n}^{\text {alg }}$ preserves (good) fibrations that are surjective on $\pi_{0}$.

Proposition 5.7. Given a space $X$ and functors $A, B$, and $C$, suppose $A(Y) \rightarrow$ $B(Y) \rightarrow C(Y)$ is a fibration sequence for all finite coproducts $Y=\bigvee X$ of $X$. If, 
on finite coproducts of $X$, either:

(1) $C$ takes connected values (Hypothesis 3.11); or

(2) $B$ and $C$ take group values (Hypothesis 13.12), and the map $B \rightarrow C$ is a surjective homomorphism of groups,

then

$$
L A_{X}(Z) \rightarrow L B_{X}(Z) \rightarrow L C_{X}(Z)
$$

is a fibration sequence for all spaces $Z$. Furthermore, the sequence is surjective on $\pi_{0}$.

Proof. This is an easy application of Theorem 4.5 .

Corollary 5.8. Under the conditions of Proposition [5.7,

$$
P_{n}^{a l g} A \rightarrow P_{n}^{a l g} B \rightarrow P_{n}^{a l g} C
$$

is a fibration and the map to the base is surjective on $\pi_{0}$.

Proof. By definition, $P_{n}^{\text {alg }} F(X)=P_{n}\left(L F_{X}\right)\left(S^{0}\right)$, so combining Proposition 5.7 with Theorem 5.1 gives the desired result.

\section{Fiber contractible implies Cartesian}

In this section, we will sketch proofs of the critical but mainly technical fact that in the cases we consider, the cross effect vanishing is equivalent to the cross effect cubes being Cartesian. We generally want to use the fact that the cross effect is contractible to conclude that the initial space in the cross-effect cube is equivalent to the (homotopy) inverse limit of the rest of the spaces. Unfortunately, this is not always true; the problem is that the homotopy fiber does not detect failure to be surjective on $\pi_{0}$. Here we show that our hypotheses on $F$ are sufficient so that this does not happen.

Lemma 6.1. Let $F$ be a functor satisfying Hypothesis 3.11 (connected values) on coproducts of $X$. If $\perp_{n} F(X) \simeq 0$, then the cube $\mathcal{C R}_{n} F(X, \ldots, X)$ defining $\perp_{n} F(X)$ is Cartesian.

Proof sketch. The first step is to consider the pullback diagram

$$
X \stackrel{p}{\rightarrow} Z \stackrel{q}{\leftarrow} Y
$$

when the spaces $X, Y$, and $Z$ are connected, and $p$ and $q$ have sections. In this case, one can directly show that $\pi_{0}$ of the homotopy inverse limit is 0 .

In general, decompose the desired inverse limit into iterated pullbacks of the form in the first step. All of the maps have sections because of the very special form of the cube $\mathcal{C R}_{n} F(X, \ldots, X)$.

This shows that the map from the initial space to the homotopy inverse limit is (trivially) surjective in $\pi_{0}$; then Cartesianness follows because the total fiber is contractible.

Lemma 6.2. Let $F$ be a functor satisfying Hypothesis 3.12 (group values) on coproducts of $X$. If $\perp_{n} F(X) \simeq 0$, then the cube $\mathcal{C} \mathcal{R}_{n} F(X)$ defining $\perp_{n} F(X)$ is Cartesian. 
Proof sketch. This strategy here is to decompose $F$ into a fibration involving $F_{0}$, the connected component of the identity, and $\pi_{0} F$, a functor to discrete groups:

$$
F_{0} \rightarrow F \rightarrow \pi_{0} F \text {. }
$$

The statement for $F_{0}$ follows from Lemma6.1. The statement for $\pi_{0} F$ is Lemma 6.3. below. Then a short argument shows no problem arises on $\pi_{0}$ in $\mathcal{C} \mathcal{R}_{n} F(X)$.

The case of discrete groups is key, so we give a complete proof for this case.

Lemma 6.3. Let $\mathcal{X}$ be an $n$-cube $(n \geq 1)$ in the category of discrete groups. If $\mathcal{X}$ has compatible sections to all structure maps (for example, $\mathcal{X}=\mathcal{C R}_{n} F(X)$ ), then the map

$$
\mathcal{X}(\emptyset) \rightarrow \lim _{U \in \mathcal{P}_{0}(\mathbf{n})} \mathcal{X}(U)
$$

is surjective.

Proof. We need to show that the map above is surjective. This is equivalent to showing that there exists an $x_{\emptyset} \in \mathcal{X}(\emptyset)$ mapping to each coherent system of elements $x_{U} \in \mathcal{X}(U)$, with $U \neq \emptyset$.

$\mathcal{X}$ is a cube of groups, and hence all of the structure maps are group homomorphisms. This allows us to subtract an arbitrary $w \in \mathcal{X}(\emptyset)$ from $x_{\emptyset}$, and subtract the images $\operatorname{Im}_{U}(w)$ of $w$ in $\mathcal{X}(U)$ from each $x_{U}$, to show that the question is equivalent to the existence of an $x_{\emptyset}-w \in \mathcal{X}(\emptyset)$ mapping to each coherent system of elements $x_{U}-\operatorname{Im}_{U}(w)$.

Given a coherent system of elements $x_{U}$ in an $n$-cube, let $w$ be the image of $x_{\{n\}}$ in $\mathcal{X}(\emptyset)$ using the section map $\mathcal{X}(\{n\}) \rightarrow \mathcal{X}(\emptyset)$. Define $z_{U}=x_{U}-\operatorname{Im}_{U}(w)$, noting that when $\{n\} \subset V$, we have $\operatorname{Im}_{V}(w)=x_{W}$, so $z_{W}=0$. By the preceding paragraph, the surjectivity that we are trying to establish is equivalent to the existence of a $z_{\emptyset}$ mapping to each coherent collection $z_{U}$.

If $n=1$, then $\lim _{U \in \mathcal{P}_{0}(\mathbf{1})} \mathcal{X}(U)=X(\{1\})$, so the section map $\mathcal{X}(\{1\}) \rightarrow \mathcal{X}(\emptyset)$ produces a $z_{\emptyset}$ mapping to $z_{\{1\}}$, as desired.

If $n>1$, then we proceed by induction, assuming the lemma is true for smaller $n$. Taking the fiber of $\mathcal{X}$ in the direction of $\{n\}$, we have an $(n-1)$-cube

$$
\mathcal{Y}(U):=\operatorname{fib}(\mathcal{X}(U) \rightarrow \mathcal{X}(U \cup\{n\})) .
$$

The cube $\mathcal{Y}$ satisfies the hypothesis of the lemma because taking fibers preserves compatible sections. Note that for $\{n\} \not \subset U$, the element $z_{U}$ passes to the fiber, since it maps to $z_{U \cup\{n\}}=0$.

Now $\mathcal{Y}$ is an $(n-1)$-cube, so by induction, the map from $\mathcal{Y}(\emptyset)$ to $\lim \mathcal{Y}(U)$ is surjective. That is, there exists a $y \in \mathcal{Y}(\emptyset)$ with $\operatorname{Im}_{U}(y)=z_{U}$. Mapping $y$ to $z \in \mathcal{X}(\emptyset)$ gives an element $z$ with $\operatorname{Im}_{U}(z)=z_{U}$ for $U \subset\{1, \ldots, n-1\}$. As above, if $\{n\} \subset U$, then $z_{U}=0$, so $\operatorname{Im}_{U}(z)=z_{U}$ in this case as well. Therefore, we have produced an element $z$ mapping to each coherent collection of elements $z_{U}$, as desired.

\section{7. $\perp$ IS A COTRIPLE}

In this section, we verify the claim that $\perp$ is actually a cotriple on the category of homotopy functors from pointed spaces to pointed spaces.

Let $\mathcal{S}$ denote the full skeletal category of finite sets whose objects are $\mathbf{k}=$ $\{1, \ldots, k\}$, for each integer $k \geq 0$. Given an integer $n$, a homotopy functor $F$, and a space $X$, we construct a functor $C$, defined on $\mathcal{S}^{\text {op }}$ and natural in $F, X$, and 
$n$, such that $C(\mathbf{k})$ is naturally isomorphic (actually equivariantly homeomorphic) to $\perp_{n}^{k} F(X)$. The functoriality of $C$ immediately shows that the iterates of $\perp_{n}$ assemble to form a simplicial object.

The difference between $\perp_{n}^{k}$ and $C(\mathbf{k})$ is the difference between computing the homotopy fiber of a cubical diagram by an iterative process and in a single step. Using a good model for the total homotopy fiber, these are isomorphic.

Since the strict commutativity of certain diagrams is essential, we begin by recalling Goodwillie's model for the homotopy fiber of a cubical diagram.

Definition 7.1 (homotopy fiber [6, Definition 1.1]). Let $I=[0,1]$ denote the unit interval. For any set $S$, let $I^{S}$ denote the product of $|S|$ copies of $I$. For clarity, when $S=\emptyset$, we take $I^{S}=\{0\}$.

Let $\mathcal{X}$ be an $S$-cube of spaces. Define the homotopy fiber of $\mathcal{X}$ to be the subspace of the space of maps

$$
\operatorname{hofib} \mathcal{X} \subset \prod_{U \subset S} \operatorname{Map}\left(I^{U}, \mathcal{X}(U)\right)
$$

with a map $\Phi \in$ hofib $\mathcal{X}$ satisfying:

(1) $\Phi$ is natural with respect to $S$,

(2) if $x=\left(x_{1}, \ldots, x_{u}\right)$ is a point in $I^{U}$ with some $x_{i}=1$, then $\Phi_{U}(x)=*$, the basepoint of $\mathcal{X}(U)$.

The utility of this definition is that computing the homotopy fiber of a cube by iterating the process of taking the fiber of maps produces a result isomorphic (that is, homeomorphic) to the result of taking the homotopy fiber in one step.

Lemma 7.2. Let $\mathcal{X}$ and $\mathcal{Y}$ be cubical diagrams of spaces, and define the cube of cubes $\mathcal{Z}=\mathcal{X} \rightarrow \mathcal{Y}$. Then there is a natural homeomorphism

$$
\text { hofib } \mathcal{Z} \stackrel{\cong}{\rightrightarrows} \text { hofib (hofib } \mathcal{X} \rightarrow \text { hofib } \mathcal{Y} \text { ). }
$$

Proof. Given the above definition of homotopy fiber, this is easy to check; it follows from the homeomorphism $\operatorname{Map}\left(I^{n}, \operatorname{Map}\left(I^{m}, X\right)\right) \cong \operatorname{Map}\left(I^{n+m}, X\right)$.

This definition of homotopy fiber has one other important property:

Lemma 7.3. Let $\mathcal{X}$ be an $S$-cube, and let 0 denote the $S$-cube that is the one point space for all $U \subset S$. Let $\mathcal{Y}=\mathcal{X} \rightarrow 0$ be the $(S \sqcup\{*\})$-cube created using the zero map to join them. Then there is a homeomorphism

$$
\operatorname{hofib} \mathcal{Y}=\operatorname{hofib}(\mathcal{X} \rightarrow 0) \stackrel{\cong}{\longrightarrow} \operatorname{hofib} \mathcal{X}
$$

induced by the inclusion of $S$ into $S \sqcup\{*\}$.

Proof. In the definition of homotopy fiber, we see that the inclusion of $S$ into $S \sqcup\{*\}$ induces a projection from

$$
\prod_{U \subset S \sqcup\{*\}} \operatorname{Map}\left(I^{U}, \mathcal{Y}(U)\right) \rightarrow \prod_{U \subset S} \operatorname{Map}\left(I^{U}, \mathcal{X}(U)\right) .
$$

The components of " $\Phi$ " corresponding to sets containing $\{*\}$ are all the constant map to the only available point, $*$, so the projection is a homeomorphism.

We now proceed with the proof that $\perp$ is a cotriple. We begin by defining a "diagonal" to encode the information needed to construct a cube of coproducts and inclusion and projection maps of the type used to define the cross effect. 
Definition 7.4 (Diagonal). For any sets $S$ and $U$, and given a function $f$ from $S$ to $U$, define the set

$$
B_{f}=\{(s, u) \mid u \neq f(s)\} \subset S \times U .
$$

Define the "diagonal" $\Delta(S, U)$ to be the element of $\mathcal{P}(S \times U)$ given by the union of all $B_{f}$ :

$$
\Delta(S, U)=\left\{B_{f} \mid f \in \operatorname{Hom}(S, U)\right\} .
$$

Since $\Delta(S, U)$ is naturally isomorphic to $\operatorname{Hom}(S, U)$ by sending $B_{f}$ to $f$, it is obviously a functor in $S$ and $U$.

Definition 7.5 (Free cube). Given sets $U$ and $S$ and a functor $g$ from the discrete category $\Delta(S, U)$ to a pointed category with coproducts (for example pointed spaces or cubes of pointed spaces), we define $\operatorname{Free}(S, U, g)$ to be the $(S \times U)$-cube $\mathcal{X}$ with vertices

$$
\mathcal{X}(A)=\bigvee_{\{B \in \Delta(S, U): A \subset B\}} g(B) .
$$

Morphisms in $\mathcal{X}$ are induced by the maps $g(B) \rightarrow g\left(B^{\prime}\right)$ that are the identity if $B=B^{\prime}$ and the zero map otherwise.

We now establish that "free cubes" are closed under the pullback operation.

Lemma 7.6. Let $m$ and $n$ be sets, let the $(m \times U)$-cube $\mathcal{X}=\operatorname{Free}(m, U, g)$ be a free cube, and let $f: n \rightarrow m$ be a function. The $(n \times U)$-cube $\mathcal{P}(f \times 1)^{*} \mathcal{X}$ is isomorphic to a free cube $\mathcal{Y}=\operatorname{Free}(n, U, h)$ with

$$
h(B)=\bigvee_{B^{\prime} \in \Delta(f, 1)^{-1}(B)} g\left(B^{\prime}\right) .
$$

Proof. This is a straightforward argument by expanding the definition of $h$ in $\mathcal{Y}(A)$, combining and interchanging the order of quantifiers to turn two coproducts into one, and then verifying that the resulting indexing set is the same as the indexing set for $\mathcal{P}(f \times 1)^{*} \mathcal{X}$.

We are now in a position to identify the relationship $\perp$ has to the free cube functor. For the rest of this section, we fix a functor $F$ and a space $X$.

Given sets $U$ and $S$, let $c_{X}$ be the function on $\Delta(S, U)$ that has a constant value $X$. Let $C(S)$ be the contravariant functor of sets $S$ given by

$$
C(S)=\operatorname{hofib} F \circ \operatorname{Free}\left(S, U, c_{X}\right) .
$$

Lemma 7.7. $C(S)$ is a contravariant functor of $S$.

Proof. Let $\mathcal{Y}=C(S)$. Given a function $f: S \rightarrow T$, we can construct the $(S \times U)$ cube $\mathcal{P}(f \times 1) * \mathcal{Y}$. The map in the indexing categories induces [3, §XI.9] a map on the homotopy fibers:

$$
\underset{\mathcal{P}(T \times U)}{\text { hofib }} F \mathcal{Y} \rightarrow \underset{\mathcal{P}(S \times U)}{\operatorname{hofib}} \mathcal{P}(f \times 1)^{*} \mathcal{Y},
$$

so it remains to construct a map

$$
\underset{\mathcal{P}(S \times U)}{\text { hofib }} F \circ \mathcal{P}(f \times 1)^{*} \mathcal{Y} \rightarrow \underset{\mathcal{P}(S \times U)}{\text { hofib }} F \circ \operatorname{Free}\left(S, U, c_{X}\right) .
$$

Recall from Lemma [.6 that $\mathcal{P}(f \times 1) * \mathcal{Y}$ is a free cube with generating function

$$
h(B)=\bigvee_{\Delta(f, 1)^{-1}(B)} X
$$


To specify the desired map between free cubes, it suffices to specify a natural transformation between their "generating functions"; the universal map from $\bigvee X$ to $X$ that is the identity on each $X$ is a natural choice in this case.

Lemma 7.8. Let $n=|U| . C(\mathbf{k})$ is equivariantly homeomorphic to $\perp_{n}^{k} F(X)$.

Proof. This is a straightforward verification that both are naturally homeomorphic (by Lemma 7.2) to the homotopy fiber of a $\mathbf{k} \times U$-cube whose vertices are

$$
F\left(\bigvee_{i=1}^{i=k} \bigvee_{v_{i} \notin V_{i}} X\right)
$$

Lemma 7.9. Let $n=|U|$. Via the homeomorphism of Lemma 7.8, the map $C(\mathbf{1}) \rightarrow$ $C(\emptyset)$ induced by $C(i: \emptyset \rightarrow \mathbf{1})$ corresponds to the map $\perp_{n} F(X) \rightarrow F(X)$ induced by $F\left(\bigvee^{n} X \rightarrow X\right)$.

Proof. This is also straightforward. Let $\mathcal{X}=\operatorname{Free}\left(\mathbf{1}, U, c_{X}\right)$ and observe that the map $\mathcal{P}(i \times 1)^{*}$ induces the identity on $\mathcal{X}(\emptyset)=\bigvee^{n} X$.

Theorem 7.10. The functor $\perp$ is a cotriple on the category of homotopy functors from pointed spaces to pointed spaces. The map $\epsilon: \perp \rightarrow 1$ is induced by the "fold map" $\bigvee X \rightarrow X$.

Proof. Lemma 7.8 identifies $\perp^{k}$ and $C(\mathbf{k})$, and Lemma 7.9 identifies the map $\epsilon$. Then the requisite identities follow from applying $C$ to the following diagrams:
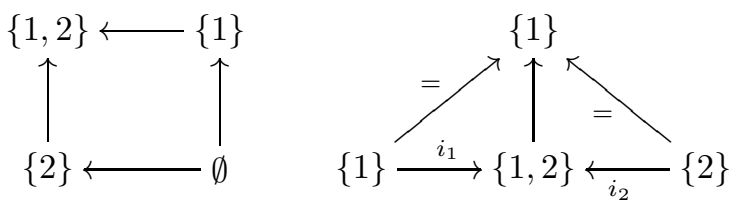

\section{Main Theorem: Outline}

To establish the Main Theorem, we use induction on $n$, beginning with the case $n=1$. We further break down the induction into "Case I", where $F$ is degree $n$; that is, $\perp_{n+1} F(X) \simeq 0$, and "Case II", which shows that Case I implies the result for arbitrary $F$. Our proof will be a ladder induction, with Case I depending on Case II for smaller values of $n$, and Case II depending on Case I for the same value of $n$.

In Section 9, we treat the case when $\perp_{n+1} F(X) \simeq 0$. In this case, we show directly that the fiber of the fibration sequence we obtain from induction,

$$
\left|\perp_{n}^{*}\left(\perp_{n} F\right)(X)\right| \rightarrow F(X) \rightarrow P_{n-1}^{\text {alg }} F(X),
$$

is a homogeneous degree $n$ functor. This implies that $F(X) \simeq P_{n}^{\text {alg }} F(X)$ in this case.

Definition 8.1 $\left(A_{F}\right)$. Define the functor $A_{F}(X)$ to be the homotopy fiber in the fibration

$$
A_{F}(X) \rightarrow\left|\perp_{n+1}^{*}\left(\perp_{n+1} F\right)(X)\right| \rightarrow F(X) .
$$


For the general case, in Section 10, we consider the auxiliary diagram:

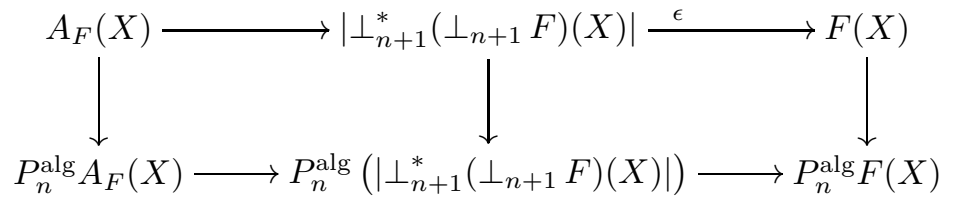

where the bottom row is shown to be a fibration sequence up to homotopy (Propositions 10.6 and 10.4). We show that $\perp_{n+1} A_{F}(X) \simeq 0$ (Lemma 10.8), and hence Case I shows that there is an equivalence of the fibers, so the square on the right is Cartesian. Then it is not hard to establish that $P_{n}^{\text {alg }}\left(\left|\perp_{n+1}^{*}\left(\perp_{n+1} F\right)(X)\right|\right) \simeq 0$ (Lemma 10.7), so that the sequence in the Main Theorem is actually a fibration sequence up to homotopy.

\section{Main Theorem, Case I: $F$ Degree $n$}

In this section, the goal is to establish that when the $(n+1)$-st cross effect of $F$ vanishes, $F$ is equivalent to its $n$-additive approximation, $P_{n}^{\text {alg }} F$. The proof of this result (Proposition 9.15) will be given in Section 9.4 .

9.1. Additivization and the bar construction. The case $n=1$ is the work of Segal.

Lemma 9.1. Suppose $F$ is a reduced functor that has either connected values (3.11) or group values (3.12) and commutes with realizations. If $\perp_{2} F \simeq 0$, then $F \simeq$ $\Omega \circ F \circ \Sigma$.

Proof. Let $X$ be a space. Under these hypotheses, the map $F(X \vee X) \stackrel{\simeq}{\rightarrow} F(X) \times$ $F(X)$ is an equivalence, so we can regard $[n] \mapsto F\left(\bigvee^{n} X\right)$ as a $\Gamma$-space. Segal's work [10, Proposition 1.4] then shows that $F(X) \simeq \Omega B F(X)$, where $B$ denotes the bar construction, and note that $B F(X) \simeq\left|F\left(X \wedge S^{1}\right)\right| \simeq F\left(S^{1} \wedge X\right)$, because $F$ commutes with realizations.

Corollary 9.2. If $F$ has either connected values (3.11) or group values (3.12), and $\perp_{n+1} F \simeq 0$, then, as a symmetric functor of $n$ variables, $\perp_{n} F$ is the infinite loop space of a symmetric functor to connective spectra $\perp_{\mathbf{n}} \mathbf{F}$ :

$$
\perp_{n} F \simeq \Omega^{\infty}\left(\perp_{\mathbf{n}} \mathbf{F}\right) .
$$

Proof. When $F$ satisfies the hypotheses above, then $\perp_{n} F$ also satisfies these hypotheses. Since $\perp_{n+1} F \simeq 0$, we know that applying $\perp_{2}$ in any input to $c r_{n} F$ is contractible (by Corollary 4.15), so Lemma 9.1 gives $\perp_{n} F$ as the first space of a connective $\Omega$-spectrum.

Corollary 9.3. Suppose $F$ is a reduced functor that commutes with realizations and has either connected values (3.11) or group values (3.12), and $\perp_{2} F \simeq 0$. Then $F \simeq P_{1} F$.

Proof. We need to show that $F \rightarrow \Omega^{n} \circ F \circ \Sigma^{n}$ is an equivalence for all $n$. This almost follows from Lemma 9.1, but one needs to check that $\Omega \circ F \circ \Sigma$ commutes with realizations. This follows from Theorem 4.5 because $F\left(S^{1} \wedge-\right)$ is always connected. 
9.2. The equivariant structure of the cross-effects of a symmetric functor. We begin with a definition of the "symmetric group" and its action on the set of integers $\mathbf{n}=\{1, \ldots, n\}$. By $\Sigma_{n}$, we mean the set of bijective set maps from $\mathbf{n}$ to $\mathbf{n}$ with the group operation corresponding to composition of functions. So no confusion arises, let us fix a representation of the symmetric group on the set $\mathbf{n}$ : let $\sigma \in \Sigma_{n}$ act on $\mathbf{n}$ by sending $j$ to $\sigma(j)$. To verify that this is an "action", note that $\tau_{*} \sigma_{*}$ sends $j$ to $\tau(\sigma(j))=(\tau \circ \sigma)(j)$, which is exactly $(\tau \sigma)_{*}$. Write $\Sigma_{n}^{+}$for the space $\Sigma_{n}$ with a disjoint basepoint added.

Definition 9.4 ([7, p. 675]). A functor of $n$ variables $H\left(X_{1}, \ldots, X_{n}\right)$ is called symmetric if for each permutation $\sigma \in \Sigma_{n}$ there is a natural transformation

$$
\sigma_{*}: H\left(X_{1}, \ldots, X_{n}\right) \rightarrow H\left(X_{\sigma^{-1}(1)}, \ldots, X_{\sigma^{-1}(n)}\right)
$$

satisfying $(\sigma \tau)_{*}=\sigma_{*} \tau_{*}$. This definition differs from Goodwillie's only in that we use $\sigma^{-1}$ where he uses $\sigma$, and consequently the action of a composition appears in a different order than his.

We immediately begin using some abbreviated notation.

Notation 9.5 $(H[\sigma])$. Given a symmetric functor of $n$ variables $H$, a map of sets $\sigma: \mathbf{n} \rightarrow \mathbf{n}$, and $n$ ambient variables $X_{1}, \ldots, X_{n}$, define

$$
H[\sigma]=H\left(X_{\sigma(1)}, \ldots, X_{\sigma(n)}\right) .
$$

With this notation, the symmetric structure is $\sigma_{*}: H[1] \rightarrow H\left[\sigma^{-1}\right]$. Note that $\tau_{*}: H\left[\sigma^{-1}\right] \rightarrow H\left[\sigma^{-1} \tau^{-1}\right]=H\left[(\tau \sigma)^{-1}\right]$.

Definition 9.6 $\left(r_{\sigma}\right)$. When $X_{1}=\cdots=X_{n}$, let $r_{\sigma}$ be the "relabeling" map induced by the isomorphisms $X_{i} \mapsto X_{\sigma(i)}$.

Definition 9.7 (Action of $\Sigma_{n}$ on the diagonal of a symmetric functor). The diagonal of a symmetric functor of $n$ variables has a natural action of the symmetric group on $n$ letters. Let $X=X_{1}=\cdots=X_{n}$, and let $\sigma \in \Sigma_{n}$. The action of $\sigma$ on $\operatorname{diag} H(X)$ is the composite

$$
H[1] \stackrel{\sigma_{*}}{\longrightarrow} H\left[\sigma^{-1}\right] \stackrel{r_{\sigma}}{\longrightarrow} H[1],
$$

where $r_{\sigma}$ is the isomorphism $X_{i} \cong X_{\sigma(i)}$ that is available because all of the $X_{i}$ are equal.

Remark 9.8. By relabeling variables, one immediately sees that the action of $\tau$ on $H[\sigma]$ is given by the composite

$$
H[\sigma] \stackrel{\tau_{*}}{\longrightarrow} H\left[\sigma \tau^{-1}\right] \stackrel{r_{\sigma \tau \sigma^{-1}}}{\longrightarrow} H[\sigma] .
$$

Example 9.9. The fundamental example is the wedge. When $n=3$, let

$$
H\left(X_{1}, X_{2}, X_{3}\right)=X_{1} \vee X_{2} \vee X_{3}
$$

The symmetric structure map for the cycle $\sigma=(123)$ in $\Sigma_{3}$ is

$$
\sigma_{*}: x_{1} \vee x_{2} \vee x_{3} \in H\left(X_{1}, X_{2}, X_{3}\right) \mapsto x_{3} \vee x_{1} \vee x_{2} \in H\left(X_{3}, X_{1}, X_{2}\right) .
$$

Note that $H\left(X_{3}, X_{1}, X_{2}\right)=H\left[\sigma^{-1}\right]$, and not $H[\sigma]$. The map $r_{\sigma}$ then has us regard $x_{3}$ as an element of $X_{1}$, etc. 
For the rest of this section, we have will work with the diagonals of symmetric functors, so set $X_{1}=\cdots=X_{n}$, but label them differently to be able to see the action of the symmetric group more clearly. To keep notation under control, we write $H$ instead of $\operatorname{diag} H$.

Lemma 9.10. Let $H$ be a symmetric functor of $n$ variables. If in each variable $i$, the categorical map

$$
H\left(\ldots, X_{i} \vee Y_{i}, \ldots\right) \stackrel{\simeq}{\longrightarrow} H\left(\ldots, X_{i}, \ldots\right) \times H\left(\ldots, Y_{i}, \ldots\right)
$$

is an equivalence, then

$$
\perp_{n} H[1] \stackrel{\simeq}{\longrightarrow} \prod_{\sigma \in \Sigma_{n}} H[\sigma] .
$$

Furthermore, with the following action, the map is equivariant.

Let $\beta \times \alpha \in \Sigma_{n} \times \Sigma_{n}$ act on the left as follows: $\alpha$ acts on $H$, and hence $\alpha$ acts on $\perp_{n} H$ (via $\perp_{n} \alpha$ ) because $\perp_{n}$ is a functor; $\beta$ acts on $\perp_{n}$ because $\perp_{n}$ is the diagonal of the symmetric functor $\mathrm{cr}_{n}$.

Let $\beta \times \alpha$ act on the right as follows: $\alpha$ acts diagonally on all copies of $H ; \beta$ acts by permuting coordinates so that the $\beta \sigma$ component of $\beta_{*}(h)$ equals the $\sigma$ component of $h$.

Proof. First note that the actions on the left commute because the action on $\perp_{n}$ is a natural transformation. The actions on the right obviously commute.

Using the hypothesis in each variable of $H$, we see that the categorical map to product (by iterating (9.1)) is an equivalence:

$$
\mathbf{H}\left(\bigvee_{i=1}^{n} X_{i}, \ldots, \bigvee_{i=1}^{n} X_{i}\right) \stackrel{\simeq}{\longrightarrow} \prod_{\sigma: \mathbf{n} \rightarrow \mathbf{n}} \mathbf{H}[\sigma]
$$

Using this to compute the cross effect, we see there is an equivalence

$$
\perp_{n} \mathbf{H}[1]=\perp_{n} \mathbf{H}\left(X_{1}, \ldots, X_{n}\right) \stackrel{\simeq}{\longrightarrow} \prod_{\sigma \in \Sigma_{n}} \mathbf{H}[\sigma] .
$$

Also, note that substituting $X_{\beta^{-1}(i)}$ for $X_{i}$ gives

$$
\perp_{n} \mathbf{H}\left[\beta^{-1}\right] \stackrel{\simeq}{\longrightarrow} \prod_{\sigma \in \Sigma_{n}} \mathbf{H}\left[\beta^{-1} \sigma\right] .
$$

A short reflection on the origins of the map in (9.2) should convince the reader that it is equivariant with respect to the action of $\Sigma_{n}$ on $H$.

The equivariance of the action of $\Sigma_{n}$ on $\perp_{n}$ is a bit more complicated, so we spell it out in detail. To orient the reader, consider the commutative diagram whose vertical composite is action by $\beta$ on $\perp_{n} H(1)$ :

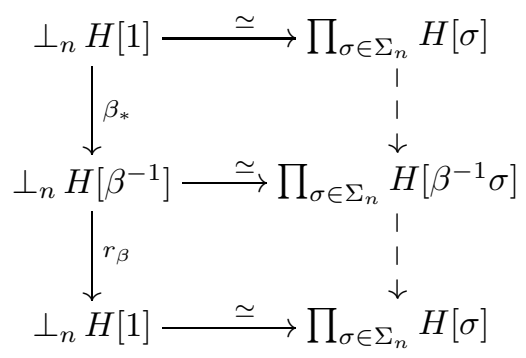


Under $\beta_{*}$, the copy of $H[\sigma]$ in the $\sigma$ component of the top row is sent to $H\left[\beta^{-1} \beta \sigma\right]$ in the $\beta \sigma$ component of the middle row. This is often a confusing point, but it is made more evident by the fact that the map $\beta_{*}$ exists even if the inputs $X_{i}$ are all different, and $\beta_{*}$ does not even require that $H$ be a symmetric functor (only that it be additive in each variable). Under the "relabeling" isomorphism $r_{\beta}$, this becomes $H[\beta \sigma]$ in the $\beta \sigma$ component of the bottom row. This shows that the action of $\beta \in \Sigma_{n}$ on $\perp_{n} H[1]$ corresponds to the action sending the $\sigma$ component of the product isomorphically to the $\beta \sigma$ component.

Lemma 9.11. Let $\mathbf{H}$ be a symmetric functor of $n$ variables taking values in spectra. There is a stable equivalence

$$
\Sigma_{n}^{+} \wedge \mathbf{H}[1] \stackrel{\simeq}{\longrightarrow} \prod_{\sigma \in \Sigma_{n}} \mathbf{H}[\sigma]
$$

given by

$$
\Sigma_{n}^{+} \wedge \mathbf{H}[1] \cong \bigvee_{\sigma \in \Sigma_{n}} \mathbf{H}[1] \stackrel{\vee r_{\sigma}}{\longrightarrow} \bigvee_{\sigma \in \Sigma_{n}} \mathbf{H}[\sigma] \hookrightarrow \simeq \prod_{\sigma \in \Sigma_{n}} \mathbf{H}[\sigma] .
$$

Let $\beta \times \alpha \in \Sigma_{n} \times \Sigma_{n}$ act on $\Sigma_{n}^{+} \wedge \mathbf{H}[1]$ as follows: $\alpha$ acts on $\mathbf{H}[1]$, and $\beta$ sends $\sigma \wedge h$ to $(\beta \sigma) \wedge h$.

With the action $\Sigma_{n} \times \Sigma_{n}$ on the right as in Lemma 9.10, this map is equivariant.

Proof. The equivariance with respect to the action of $\beta$ is immediate. The equivariance with respect to the action of $\alpha$ follows from the commutativity of both of the following squares, where the vertical composites are the action of $\alpha$ on the left and one component of the right:

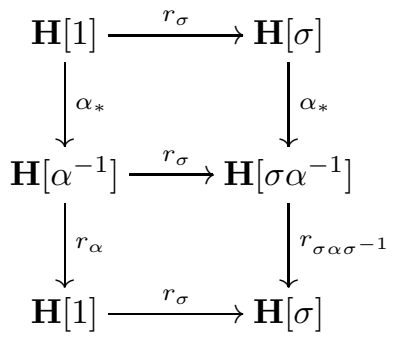

The map $r_{\sigma \alpha \sigma^{-1}}$ is used for the action of $\alpha$ on $H[\sigma]$, as noted in Remark 9.8. The stable equivalence of coproducts and products is the last map used.

Corollary 9.12. Let $\mathbf{H}$ be a symmetric functor of $n$ variables taking values in spectra. If the second cross effect in each variable of $\mathbf{H}$ is contractible, then there is a weak equivariant map

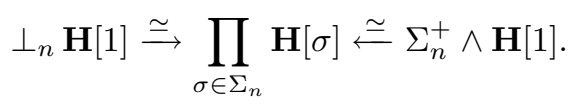

The action of $\beta \in \Sigma_{n}$ on $\perp_{n}$ corresponds to multiplication by $\beta$ on $\Sigma_{n}^{+}$on the right side of the equation, and the action on $\mathbf{H}$ is the same on both sides.

Proof. Since $\mathbf{H}$ is a functor to spectra, the vanishing of the second cross effect implies that the hypotheses of Lemma 9.10 are satisfied. Combining Lemmas 9.10 and 9.11 gives the desired result. 
This type of identification goes back at least to Eilenberg and Mac Lane in the 1950s [4, Theorem 9.1]. With this model, the map $\epsilon: \perp_{n} \mathbf{H} \rightarrow \mathbf{H}$ is given by $\sigma \wedge x \mapsto x$.

Corollary 9.13. Let $\bar{\epsilon}$ be the map $\Sigma_{n}^{+} \wedge \mathbf{H}[1] \rightarrow \mathbf{H}[1]$ sending $\sigma \wedge h$ to $h$. Under the conditions of Corollary 9.12 , the following diagram commutes:

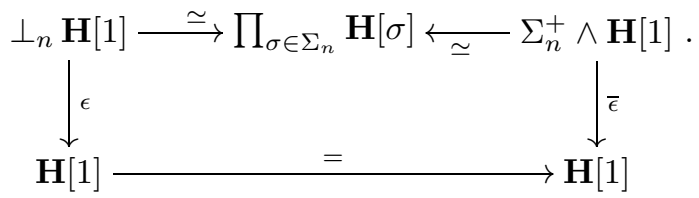

Proof. Let $X=X_{1}=\cdots=X_{n}$ be spaces. The map $\epsilon$ is induced by the fold map $\bigvee X_{i} \rightarrow X$, so it sends

$$
\mathbf{H}[\sigma]=\mathbf{H}\left(X_{\sigma(1)}, \ldots, X_{\sigma(n)}\right) \rightarrow \mathbf{H}(X, \ldots, X)=\mathbf{H}\left(X_{1}, \ldots, X_{n}\right)=\mathbf{H}[1]
$$

by the isomorphism $X_{\sigma(i)}=X=X_{i}$; this is the map we have denoted $r_{\sigma^{-1}}$. On $\sigma \wedge h$, the composite of the upper and left maps is then $r_{\sigma^{-1}} r_{\sigma}(h)=h$, and $\bar{\epsilon}(\sigma \wedge h)=h$, so the diagram commutes.

\subsection{Iterated cross effects produce homogeneous functors.}

Lemma 9.14. If $F$ has either connected values (3.11) or group values (3.12) on coproducts of $X$, and $\perp_{n+1} F(X) \simeq 0$, then

$$
\left|\perp_{n}^{*}\left(\perp_{\mathbf{n}} \mathbf{F}\right)(X)\right| \simeq \perp_{\mathbf{n}} \mathbf{F}(X) \wedge_{\Sigma_{n}} E \Sigma_{n}^{+},
$$

where $\perp_{\mathbf{n}} \mathbf{F}$ denotes the lift to spectra of $\perp_{n} F$, as in Corollary 9.2

With these hypotheses, Corollary 9.2 shows that $\perp_{n} F(X) \simeq \Omega^{\infty} \perp_{\mathbf{n}} \mathbf{F}(X)$, so we are entitled to consider the functor to spectra " $\perp_{\mathbf{n}} \mathbf{F}$ ". Corollary 4.15 shows that the second cross effect in each variable of $\perp_{\mathbf{n}} \mathbf{F}$ is contractible, so we may apply Corollary 9.12 with $\mathbf{H}=\perp_{\mathbf{n}} \mathbf{F}$ to conclude that

$$
\perp_{n} \perp_{\mathbf{n}} \mathbf{F}(X) \simeq \Sigma_{n}^{+} \wedge \perp_{\mathbf{n}} \mathbf{F}(X) .
$$

We are now in a position to understand $\perp_{n}^{*} \perp_{\mathbf{n}} \mathbf{F}$. The issue of how multiplication by $\sigma$ arises from the $\epsilon$ above is somewhat subtle, so we spell it out in detail. Applying (9.3) repeatedly at each level, we have

$$
\perp_{n}^{k} \perp_{\mathbf{n}} \mathbf{F}(X) \simeq \underbrace{\sum_{n}^{+} \wedge \cdots \wedge \Sigma_{n}^{+}}_{k \text { factors }} \wedge \perp_{\mathbf{n}} \mathbf{F}(X) .
$$

Recall that the face maps from dimension $n$ to $n-1$ are given by $d_{i}=\perp_{n}^{i} \epsilon \perp_{n}^{n-i}$. In dimension $k$, the face map $d_{k}=\epsilon \perp_{n}^{k}$ just drops the first element (by Corollary 9.13):

$$
d_{k}\left(g_{k} \wedge \cdots \wedge g_{1} \wedge y\right)=g_{k-1} \wedge \cdots \wedge g_{1} \wedge y .
$$

To compute the others, note that for any $f$, the map $\perp_{n}(f)$ is equivariant with respect to the action of $\Sigma_{n}$ on $\perp_{n}$ (by permuting inputs), so in particular $\perp_{n}(\epsilon)$ : $\perp_{n}\left(\perp_{n} F\right) \rightarrow \perp_{n} F$ is equivariant with respect to the action on of $\Sigma_{n}$ on the leftmost $\perp_{n}$. Therefore,

$$
\begin{aligned}
\perp_{n} \epsilon(g \wedge y) & =\perp_{n} \epsilon(g *(1 \wedge y)) \\
& =g * \perp_{n} \epsilon(1 \wedge y) \\
& =g * y
\end{aligned}
$$


where the last follows since the degeneracy $\delta: \perp_{n} F \rightarrow \perp_{n}^{2} F$, which has $\delta(y)=1 \wedge y$, is a section to the face map $\perp_{n} \epsilon$. This argument shows that all of the face maps $d_{j}$ with $0 \leq j<k$ are given by multiplying $g_{j+1}$ by the next coordinate to the right (either $g_{j}$ if $j>0$ or $y$ if $j=0$ ).

This is a standard model for $E \Sigma_{n}^{+} \wedge_{\Sigma_{n}} \perp_{\mathbf{n}} \mathbf{F}(X)$, so we have proven Lemma 9.14

\subsection{Proof of Main Theorem, Case I.}

Proposition 9.15. Suppose that $F$ commutes with realizations and has connected values (3.11) or group values (3.12). If $\perp_{n+1} F \simeq 0$, then $F \simeq P_{n} F$.

Proof. When $n=0$, the hypothesis that $F$ is reduced makes the result trivial. When $n=1$, Corollary 9.3 shows that $F(X) \simeq P_{1} F(X)$, which establishes the truth of the base case in our induction.

Finally, when $n>1$ we apply Proposition 10.9 with one smaller $n$ to produce a fibration sequence

$$
\left|\perp_{n}^{*}\left(\perp_{n} F\right)\right| \rightarrow F \rightarrow P_{n-1} F,
$$

where the map $F \rightarrow P_{n-1} F$ is surjective on $\pi_{0}$. (Recall that since $F$ commutes with realizations, $P_{n-1}^{\text {alg }} F \simeq P_{n-1} F$.)

We now show that the fiber here is an $n$-excisive functor. From Corollary 9.2 , we know that $\perp_{n} F$ lifts to a functor to connective spectra $\perp_{\mathbf{n}} \mathbf{F}$, so that $\perp_{n} F \simeq$ $\Omega^{\infty} \perp_{\mathbf{n}} \mathbf{F}$. Using this, we have

$$
\left|\perp_{n}^{*}\left(\perp_{n} F\right)\right| \simeq\left|\perp_{n}^{*}\left(\Omega^{\infty} \perp_{\mathbf{n}} \mathbf{F}\right)\right| .
$$

The functor $\Omega^{\infty}$ is a right adjoint, so it preserves homotopy fibers, and hence commutes with $\perp_{n}$ :

$$
\left|\perp_{n}^{*}\left(\Omega^{\infty} \perp_{\mathbf{n}} \mathbf{F}\right)\right| \simeq\left|\Omega^{\infty} \perp_{n}^{*}\left(\perp_{\mathbf{n}} \mathbf{F}\right)\right| .
$$

Now $\perp_{\mathbf{n}} \mathbf{F}$ is a functor to connective spectra, and hence all applications of $\perp_{n}$ to it result in functors to connective spectra, so we can use 1 to move $\Omega^{\infty}$ outside of the realization:

$$
\left|\Omega^{\infty} \perp_{n}^{*}\left(\perp_{\mathbf{n}} \mathbf{F}\right)\right| \simeq \Omega^{\infty}\left|\perp_{n}^{*}\left(\perp_{\mathbf{n}} \mathbf{F}\right)\right| .
$$

Then Lemma 9.14 shows that the term inside the realization computes the homotopy orbits of the $\Sigma_{n}$ action on $\perp_{\mathbf{n}} \mathbf{F}$ :

$$
\left|\perp_{n}^{*}\left(\perp_{\mathbf{n}} \mathbf{F}\right)\right| \simeq \perp_{\mathbf{n}} \mathbf{F} \wedge_{\Sigma_{n}} E \Sigma_{n}^{+} .
$$

Combining all of these, we identify the fiber in (9.4) as the infinite loop space of the preceding homotopy orbit spectrum:

$$
\left|\perp_{n}^{*}\left(\perp_{n} F\right)\right| \simeq \Omega^{\infty}\left(\perp_{\mathbf{n}} \mathbf{F} \wedge_{\Sigma_{n}} E \Sigma_{n}^{+}\right) .
$$

We now establish that this functor is $n$-excisive. Since it is known to be the fiber of $F \rightarrow P_{n-1} F$, this will imply that it is actually homogeneous $n$-excisive.

The functor $\Omega^{\infty}$ preserves Cartesian squares, so we need only establish that $\perp_{\mathbf{n}} \mathbf{F} \wedge_{\Sigma_{n}} E \Sigma_{n}^{+}$is $n$-excisive. By hypothesis, $\perp_{n+1} F \simeq *$, so Corollary 4.15 shows that the second cross effect in any variable of $\mathbf{c r}_{\mathbf{n}} \mathbf{F}$ is contractible. Hence, by Corollary 9.3, in each variable $\mathbf{c r}_{\mathbf{n}} \mathbf{F}$ is 1-excisive. Then [6, Proposition 3.4] shows 
that its diagonal, $\perp_{\mathbf{n}} \mathbf{F}$, is $n$-excisive. That is, given any strongly co-Cartesian $(n+1)$-cube $\mathcal{X}$, the map

$$
\perp_{\mathbf{n}} \mathbf{F} \mathcal{X}(\emptyset) \rightarrow \operatorname{holim}_{\mathcal{P}_{0}(\mathbf{n}+\mathbf{1})} \perp_{\mathbf{n}} \mathbf{F} \mathcal{X}
$$

is an equivalence. Since $\Sigma_{n}$ acts naturally on $\perp_{\mathbf{n}} \mathbf{F}$, this is a $\Sigma_{n}$-equivariant map, so it is still an equivalence after taking homotopy orbits. This establishes that the functor $\perp_{\mathbf{n}} \mathbf{F} \wedge_{\Sigma_{n}} E \Sigma_{n}^{+}$is $n$-excisive, as desired.

Equation (9.4) is a fibration sequence up to homotopy, so we know the natural map

$$
\left|\perp_{n}^{*}\left(\perp_{n} F\right)\right| \rightarrow \operatorname{fib}\left(F \rightarrow P_{n-1} F\right)
$$

is an equivalence. Applying $P_{n}$ and using the fact that we have just shown that $\left|\perp_{n}^{*}\left(\perp_{n} F\right)\right|$ is $n$-excisive, we have

$$
\begin{aligned}
\left|\perp_{n}^{*}\left(\perp_{n} F\right)\right| & \simeq P_{n}\left|\perp_{n}^{*}\left(\perp_{n} F\right)\right| \\
& \simeq P_{n} \operatorname{fib}\left(F \rightarrow P_{n-1} F\right) \\
& \simeq \operatorname{fib}\left(P_{n} F \rightarrow P_{n-1} F\right) \\
& \simeq D_{n} F .
\end{aligned}
$$

When $F$ commutes with realizations, the map $F \rightarrow P_{n-1} F$ is surjective on $\pi_{0}$ (Theorem 5.1). This lets us argue that the total space $F$ of the fibration in (9.4) is $n$-excisive, since the base and the fiber are. The argument is straightforward but does require a variant of the five-lemma on $\pi_{0}$ to make a conclusion about $\pi_{0} F$. Once we know $F$ is $n$-excisive, we know $F \simeq P_{n} F$, as desired.

Corollary 9.16. If $F$ is a reduced functor that has either connected values (3.11) or group values (3.12) on coproducts of $X$, and $\perp_{n+1} F(X) \simeq 0$, then $F(X) \simeq$ $P_{n}^{a l g} F(X)$.

Proof. $P_{n}^{\text {alg }} F(X)$ is defined by evaluating the functor $P_{n}\left(L F_{X}\right)$ at the space $S^{0}$, and $L F_{X}$ commutes with realizations (by Lemma 3.6), so by Proposition 3.10, we have $P_{n}\left(L F_{X}\right)\left(S^{0}\right) \simeq P_{n} F(X)$. Hence the result follows from Proposition 9.15 ,

\section{Main Theorem, Case II: General $F$}

In this section, the goal is to establish the other side of the "ladder induction" for Theorem 3.13. We proceed essentially as outlined in Section 8 . We actually decompose the problem further, considering functors to discrete groups or connected spaces. As one might expect, the case of a functor to discrete groups is the pivotal one.

10.1. Functors to discrete groups: $\perp G^{a b}=0$. This section shows that a certain functor $G_{n}^{a b}$ has no $(n+1)^{\text {st }}$ cross effect, as one would expect in view of the construction of $G_{n}^{a b}$ (given below). In this section, we consider a functor $G$ from spaces to discrete groups (for example, $G(X)=\pi_{0} \Omega(X)$ ).

Definition 10.1. Given an $n>0$ and a functor $G$ to discrete groups, define $G_{n}^{\prime}:=\operatorname{Im}\left(\epsilon: \perp_{n+1} G \rightarrow G\right)$ and $G_{n}^{a b}:=\operatorname{coker}(\epsilon)$. Usually, the $n$ is clear from context, and we will abbreviate these $G^{\prime}$ and $G^{a b}$. 
There is a short exact (fibration) sequence of groups

$$
G^{\prime}(X) \rightarrow G(X) \rightarrow G^{a b}(X)
$$

and this sequence is surjective on $\pi_{0}$ (i.e., right exact).

Remark 10.2. Note that since $\perp_{n+1} G$ is constructed by taking a kernel, the image of $G^{\prime}$ is normal in $G$.

Our motivation for the preceding notation comes from considering the case when the source and target category under consideration are both the category of groups, and the functor $G$ is the identity $G(H)=H$. In this case, the image of $\perp_{2} G(H)$ in $G(H)$ is the first derived subgroup of $H$. The cokernel of the map $\perp_{2} G(H) \rightarrow G(H)$ is the abelianization, $H^{a b}$. Lacking a more appropriate name for modding out by higher derived subgroups, we continue to use the same notation in that case.

Lemma 10.3. If $F$ takes values in discrete groups, then with $G^{\prime}$ and $G^{a b}$ as in Definition 10.1, $\perp G^{a b}(X) \simeq 0$.

Proof. From the construction of $G^{\prime}$, the map $\epsilon: \perp G \rightarrow G$ factors through the inclusion $i: G^{\prime} \rightarrow G$. Applying $\perp$ again results in the following diagram:

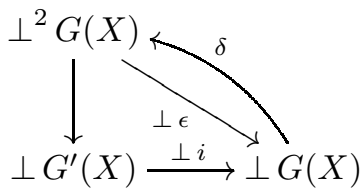

The map $\perp \epsilon$ has a section, $\delta$, so it is surjective. Hence $\perp i$ is also surjective. Consider the short exact sequence of functors to discrete groups in Equation (10.1) defining $G^{a b}$. If we show that $\perp$ preserves this short exact sequence, then the surjectivity of the map $\perp i$ will imply that $\perp G^{a b}=0$.

Short exact sequences of discrete groups are fiber sequences that are surjective on the base space. The functor $\perp$ preserves fiber sequences because the construction involves only taking fibers. The functor $\perp$ preserves surjections because all of the maps in the cube $\mathcal{C R}_{n+1} F(X, \ldots, X)$ defining $\perp_{n+1} F(X)$ have sections, and hence taking fibers with respect to them does not lower connectivity.

10.2. $P_{n}^{\text {alg }}$ preserves $A_{F}$ fibration. This section establishes that $P_{n}^{\text {alg }}$ actually produces a fibration when applied to the fibration defining $A_{F}$. The results in this section also contain a statement about the map $F \rightarrow P_{n}^{\text {alg }} F$, because in the case of $F$ taking values in discrete groups, the proof that this map is surjective on $\pi_{0}$ uses the same technical details as the proof that we get a fibration.

To remind the reader that the functor takes values in discrete groups in the next proposition, we use the letter $G$ (for group) to denote the functor, instead of the usual $F$.

Proposition 10.4. If $G$ takes values in discrete groups (so in particular $G$ satisfies Hypothesis 3.12), then the following is a fibration sequence up to homotopy:

$$
P_{n}^{a l g} A_{G}(X) \rightarrow P_{n}^{a l g}\left(\left|\perp_{n+1}^{*+1} G(X)\right|\right) \rightarrow P_{n}^{a l g} G(X) .
$$

Proof. Replacing the base $G$ in the definition of $A_{G}$ (Equation (8.1)) with $G^{\prime}$ from Definition 10.1, we have the fibration sequence

$$
A_{G}(X) \rightarrow\left|\perp_{n+1}^{*+1} G(X)\right| \rightarrow G^{\prime}(X),
$$


and this sequence is surjective on $\pi_{0}$. The hypotheses of Corollary 5.8 are satisfied by the sequences in (10.1) and (10.3), so applying $P_{n}^{\text {alg }}$ both are fibration sequences whose maps to the base spaces are surjective on $\pi_{0}$ :

$$
\begin{gathered}
P_{n}^{\mathrm{alg}} G^{\prime}(X) \rightarrow P_{n}^{\mathrm{alg}} G(X) \rightarrow P_{n}^{\mathrm{alg}} G^{a b}(X), \\
P_{n}^{\mathrm{alg}}\left(A_{G}\right)(X) \rightarrow P_{n}^{\mathrm{alg}}\left(\left|\perp_{n+1}^{*+1} G(-)\right|\right)(X) \rightarrow P_{n}^{\mathrm{alg}} G^{\prime}(X) .
\end{gathered}
$$

The aim now is to show that (10.5) remains a fibration when the base $P_{n}^{\text {alg }} G^{\prime}(X)$ is replaced by $P_{n}^{\text {alg }} G(X)$. From Lemma 10.3. $\perp_{n+1} G^{a b}(X) \simeq 0$, so Corollary 9.16 shows that $P_{n}^{\text {alg }} G^{a b}(X) \simeq G^{a b}(X)$, which is a discrete space. Then, using the long exact sequence on homotopy, the fibration in (10.4) gives $P_{n}^{\text {alg }} G^{\prime}(X) \stackrel{\simeq}{\longrightarrow} P_{n}^{\text {alg }} G(X)$ except possibly on $\pi_{0}$, where the map is injective. This is enough to show that changing the base in (10.5) from $P_{n}^{\text {alg }} G^{\prime}(X)$ to $P_{n}^{\text {alg }} G(X)$ still yields a fibration. That is, (10.2) is a fibration (but perhaps not surjective on $\pi_{0}$ ).

Proposition 10.5. If $G$ takes values in discrete groups (so in particular $G$ satisfies Hypothesis 3.12 , , then

$$
\pi_{0} P_{n}^{a l g} G(X) \cong \operatorname{coker}_{G p s}\left(\pi_{0} \epsilon\right),
$$

and the map $\pi_{0} G \rightarrow \pi_{0} P_{n}^{a l g} G$ is the universal map to the cokernel of $\pi_{0} \epsilon$ in the category of groups.

Proof. As in the preceding Proposition 10.4, we have the following fibration sequence that is surjective on $\pi_{0}$ :

$$
P_{n}^{\mathrm{alg}}\left(A_{G}\right)(X) \rightarrow P_{n}^{\mathrm{alg}}\left(\left|\perp_{n+1}^{*+1} G(-)\right|\right)(X) \rightarrow P_{n}^{\mathrm{alg}} G^{\prime}(X) .
$$

Lemma 10.7 shows that the total space in this fibration is contractible, and the map to the base is surjective on $\pi_{0}$, so $\pi_{0} P_{n}^{\text {alg }} G^{\prime}(X)=0$.

Also following Proposition 10.4, we have the following diagram in which the horizonal rows are fibrations that are surjective on $\pi_{0}$ :

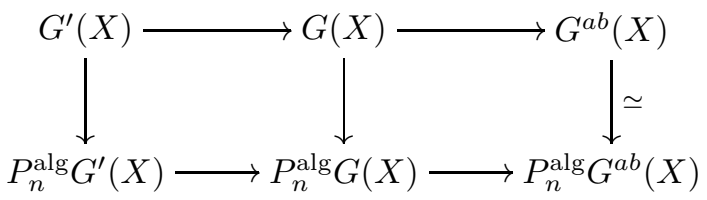

Since $\pi_{0} P_{n}^{\text {alg }} G^{\prime}(X)=0$, the long exact sequence for the bottom fibration implies that $\pi_{0} P_{n}^{\text {alg }} G(X) \cong \pi_{0} P_{n}^{\text {alg }} G^{a b}$. The right-hand vertical map is an equivalence, again as noted in the preceding proposition, using Lemma 10.3 and Corollary 9.16, Combining these, we have

$$
\begin{aligned}
\pi_{0} P_{n}^{\text {alg }} G(X) & \cong \pi_{0} P_{n}^{\text {alg }} G^{a b}(X) \\
& \cong \pi_{0} G^{a b}(X),
\end{aligned}
$$

which is isomorphic to $\operatorname{coker}_{G p s}\left(\pi_{0} \epsilon\right)$ because the map $\epsilon: \perp_{n+1} G(X) \rightarrow G(X)$ factors through $G^{\prime}(X)$.

Proposition 10.6. If $F$ has connected values (3.11), then the following is a fibration sequence up to homotopy:

$$
P_{n}^{a l g} A_{F}(X) \rightarrow P_{n}^{a l g}\left(\left|\perp_{n+1}^{*+1} F(X)\right|\right) \rightarrow P_{n}^{a l g} F(X),
$$

and furthermore the map $F(X) \rightarrow P_{n}^{\text {alg }} F(X)$ is (trivially) surjective on $\pi_{0}$. 
Proof. If $F$ has connected values on coproducts of $X$, then

$$
A_{F}(X) \rightarrow\left|\perp_{n+1}^{*+1} F(X)\right| \rightarrow F(X)
$$

is a fibration over a connected base. Therefore, by Corollary 5.8, applying $P_{n}^{\text {alg }}$ yields a fibration, so Equation (10.2) is a fibration.

The map $0 \rightarrow F$ is 0 -connected, so Theorem 5.1 shows that $0 \simeq P_{n}^{\text {alg }}(0) \rightarrow$ $P_{n}^{\text {alg }} F(X)$ is 0 -connected as well. Hence $\pi_{0} P_{n}^{\text {alg }} F(X)=0$.

10.3. If $m<n$, then $P_{n}^{\text {alg }}\left|\perp_{n}^{*}\left(\perp_{n} F\right)\right| \simeq 0$. This section establishes the relatively easy fact that for $\perp_{n}$, the part of the Goodwillie tower below degree $n$ is trivial.

Lemma 10.7. Let $R\left(X_{1}, \ldots, X_{n}\right)=\left|c r_{n}\left(\perp_{n}^{*} F\right)\left(X_{1}, \ldots, X_{n}\right)\right|$ be a functor of $n$ variables. Define the diagonal of such a functor to be the functor of one variable given by $(\operatorname{diag} R)(X)=R(X, \ldots, X)$. Then $P_{m}^{\text {alg }}(\operatorname{diag} R)(X) \simeq 0$ for $0 \leq m<n$.

Proof. Two results in Goodwillie's work [7, Lemmas 3.1 and 3.2] combine to show that if $H\left(X_{1}, \ldots, X_{n}\right)$ is a functor of $n$ variables that is contractible whenever some $X_{i}$ is contractible (this is called a "multi-reduced" functor), then $P_{m}(\operatorname{diag} H) \simeq 0$ for $0 \leq m<n$. Writing out $P_{m}^{\operatorname{alg}}(\operatorname{diag} R)(X)=P_{m}\left[L(\operatorname{diag} R)_{X}\right]\left(S^{0}\right)$, it is easy to check that $L(\operatorname{diag} R)_{X}$ is the diagonal of a multi-reduced functor, so Goodwillie's result applies.

10.4. The functor $A_{F}$ has no $n+1$ cross effect. Having created the functor $A_{F}$ to be " $F$ with the cross effect killed", we now need to establish that $\perp A_{F} \simeq 0$. The main issue is the commuting of the $\perp$ and the realization.

Lemma 10.8. Let $F$ be a functor that has either connected values (3.11) or group values (3.12), let $\perp$ denote $\perp_{n}$ for some $n$, and let $A_{F}$ be the functor given in Definition 8.1. Then $\perp A_{F}$ is contractible.

Proof. Taking cross effects is a homotopy inverse limit construction, and homotopy inverse limits commute, so

$$
\perp A_{F} \simeq \text { hofib }\left(\perp\left|\perp^{*+1} F\right| \rightarrow \perp F\right) .
$$

It is easy to check that if $F$ has connected values, then so does $\perp F$, and also $\left|\perp^{*+1} F\right|$. If $F$ has group values, then so does $\perp F$; hence $\perp^{*+1} F$ is a functor to $\operatorname{Simp}(\mathcal{G})$ - simplicial grouplike $H$-spaces. As remarked prior to Hypothesis 3.12 . the rigidity of our definition of $H$-space implies the realization of $\perp^{*+1} F$ is still a functor to $\mathcal{G}$; that is, $\left|\perp^{*+1} F\right|$ has group values. By Lemma 4.10, $\perp$ commutes with the realization in that functor, so $\perp\left|\perp^{*}(\perp F)\right| \simeq\left|\perp \perp^{*}(\perp F)\right|$. Finally, the existence of $\delta: \perp F \rightarrow \perp^{2} F$ shows that $\perp F$ is the augmentation of the simplicial space $\perp \perp^{*}(\perp F)$, so the standard "extra degeneracy" argument [11, Exercise 8.4.6, p. 275] shows that $\left|\perp \perp^{*}(\perp F)\right| \simeq \perp F$, and hence that

$$
A_{F} \simeq \operatorname{hofib}(\perp F \rightarrow \perp F) \simeq 0 \text {, }
$$

as desired.

\subsection{Proof of Main Theorem, Case II.}

Proposition 10.9. If $F$ is a reduced functor that has either connected values (3.11) or group values (3.12) on coproducts of $X$, then the following is a fibration sequence up to homotopy:

$$
\left|\perp_{n+1}^{*}\left(\perp_{n+1} F\right)(X)\right| \stackrel{\epsilon}{\rightarrow} F(X) \rightarrow P_{n}^{a l g} F(X) .
$$


Furthermore, the map $\pi_{0} F(X) \rightarrow \pi_{0} P_{n}^{\text {alg }} F(X)$ is the universal map to the cokernel of the group homomorphism $\pi_{0} \perp_{n+1} F(X) \rightarrow \pi_{0} F(X)$.

Proof. First, suppose that $F(X)$ takes either connected values or discrete group values on coproducts of $X$. Consider the auxiliary diagram created by applying $P_{n}^{\text {alg }}$ to the fibration sequence defining $A_{F}(X)$ :

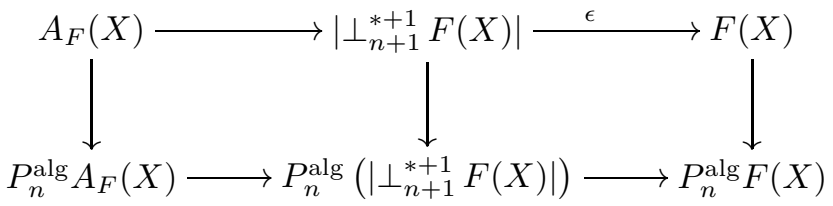

Proposition 10.6 (in the case of connected values) or Proposition 10.4 (in the case of discrete group values) shows that the bottom row is a fibration sequence up to homotopy. Proposition 10.6 (connected values) or Proposition 10.5 (discrete group values) imply that the map $F(X) \rightarrow P_{n}^{\text {alg }} F(X)$ is surjective on $\pi_{0}$. Lemma 10.8 shows that $\perp_{n+1} A_{F}(X) \simeq 0$, so that Corollary 9.16 gives $A_{F}(X) \simeq P_{n}^{\text {alg }} A_{F}(X)$, and hence the square on the right is homotopy Cartesian. Lemma 10.7 shows that $P_{n}^{\text {alg }}\left(\left|\perp_{n+1}^{*+1} F(X)\right|\right) \simeq 0$, so this square being Cartesian is equivalent to (10.6) being a fibration sequence up to homotopy, as we wanted to establish.

We can reduce the general problem when $F$ has group values to the cases of connected and discrete group values that we have already considered by examining the fibration

$$
\widehat{F}(X) \rightarrow F(X) \rightarrow \pi_{0} F(X),
$$

where $\widehat{F}(X)$ is the component of the basepoint in $F(X)$. This gives rise to the following square:

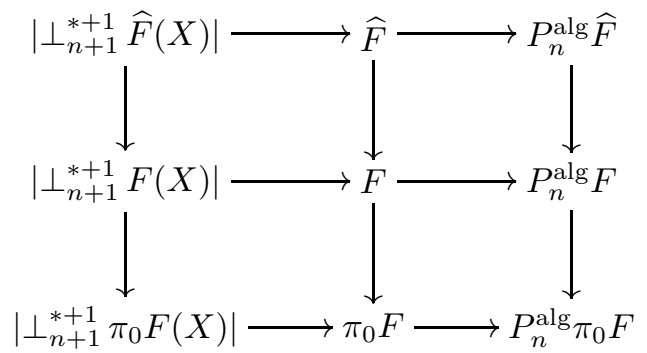

It is straightforward to check that every row and column except the middle row is a fibration that is surjective in $\pi_{0}$, and that the composition of the two maps in the middle is null homotopic, and we have shown that the map $F \rightarrow P_{n}^{\text {alg }} F$ is surjective on $\pi_{0}$. This gives us the data required to use the $3 \times 3$ lemma for fibrations to show that the middle row (i.e., (10.2) ) is a fibration and surjective on $\pi_{0}$.

The statement about $\pi_{0}$ is trivial in the connected case; $\pi_{0}$ of every space in the top row is zero (which is trivially a group). This implies that the vertical arrows connecting the second and third rows are $\pi_{0}$-isomorphisms, so the statement about $\pi_{0}$ follows from Proposition 10.5 .

\section{ACKNOWLEDGEMENTS}

This paper is based on my Ph.D. thesis at the University of Illinois. I thank Randy McCarthy for his guidance. Thanks also to Jim McClure, Tom Goodwillie, and the referee for their helpful suggestions. 


\section{REFERENCES}

1. Jon Beck, Classifying spaces for homotopy-everything H-spaces, H-spaces (Actes Réunion Neuchâtel, 1970), Springer, Berlin, 1971, pp. 54-62. Lecture Notes in Math., Vol. 196. MR $0292073(45: 1160)$

2. A. K. Bousfield and E. M. Friedlander, Homotopy theory of $\Gamma$-spaces, spectra, and bisimplicial sets, Geometric applications of homotopy theory II (Proc. Conf., Evanston, Ill., 1977), Springer, Berlin, 1978, pp. 80-130. MR0513569 (80e:55021)

3. A. K. Bousfield and D. M. Kan, Homotopy limits, completions and localizations, SpringerVerlag, Berlin, 1972, Lecture Notes in Mathematics, Vol. 304. MR0365573 (51:1825)

4. Samuel Eilenberg and Saunders Mac Lane, On the groups $H(\Pi, n)$. II. Methods of computation, Ann. of Math. (2) 60 (1954), 49-139. MR0065162 (16:391a)

5. Thomas G. Goodwillie, Calculus. I. The first derivative of pseudoisotopy theory, K-Theory 4 (1990), no. 1, 1-27. MR.1076523 (92m:57027)

6. Calculus. II. Analytic functors, K-Theory 5 (1991/92), no. 4, 295-332. MR.1162445 (93i:55015)

7. , Calculus. III. Taylor series, Geometry \& Topology 7 (2003), 645-711. MR2026544 (2005e:55015)

8. Brenda Johnson and Randy McCarthy, Taylor towers for functors of additive categories, J. Pure Appl. Algebra 137 (1999), no. 3, 253-284. MR1685140 (2000b:18018)

9. _ Deriving calculus with cotriples, Trans. Amer. Math. Soc. 356 (2004), 757-803. MR2022719

10. Graeme Segal, Categories and cohomology theories, Topology 13 (1974), 293-312. MR0353298 (50:5782)

11. Charles A. Weibel, An introduction to homological algebra, Cambridge Studies in Advanced Mathematics, vol. 38, Cambridge University Press, Cambridge, 1994. MR1269324 (95f:18001)

Department of Mathematics, Northwestern University, Evanston, Illinois 60208

E-mail address: amauer@math.northwestern.edu 